University of Rhode Island

DigitalCommons@URI

Open Access Master's Theses

2020

\title{
ACOUSTIC AND PERCEPTUAL OUTCOMES OF VOCAL TRAINING FOR TRANSGENDER WOMEN
}

Justin Yehle

University of Rhode Island, justinyehle@my.uri.edu

Follow this and additional works at: https://digitalcommons.uri.edu/theses

\section{Recommended Citation}

Yehle, Justin, "ACOUSTIC AND PERCEPTUAL OUTCOMES OF VOCAL TRAINING FOR TRANSGENDER WOMEN" (2020). Open Access Master's Theses. Paper 1829.

https://digitalcommons.uri.edu/theses/1829

This Thesis is brought to you for free and open access by DigitalCommons@URI. It has been accepted for inclusion in Open Access Master's Theses by an authorized administrator of DigitalCommons@URI. For more information, please contact digitalcommons-group@uri.edu. 
ACOUSTIC AND PERCEPTUAL OUTCOMES OF VOCAL TRAINING FOR

TRANSGENDER WOMEN

BY

JUSTIN YEHLE

A THESIS SUBMITTED IN PARTIAL FULFULMENT OF THE

REQUIREMENTS FOR THE DEGREE OF

MASTER OF SCIENCE

IN

SPEECH-LANGUAGE PATHOLOGY

UNIVERSTY OF RHODE ISLAND

2020 


\section{MASTER OF SCIENCE THESIS}

OF

\section{JUSTIN YEHLE}

\section{APPROVED:}

Thesis Committee:

Major Professor: Leslie Mahler

Rachel Smith

Natalie Sabik

Nassar H. Zawia

DEAN OF THE GRADUATE SCHOOL 


\begin{abstract}
Purpose: This study investigated the acoustic and perceptual outcomes of a specific six-week voice training protocol for transgender women, by analyzing acoustic features of speech and voice. The authors sought to establish statistical and clinical significance between speaking fundamental frequency and self-perceptual ratings of communicative experiences, pre- and post-training.

Methods: Speech samples were collected from 10 transgender women. Acoustic variables analyzed for this research include: fundamental frequency (F0) of vowel prolongation, speaking fundamental frequency (SFF) of spontaneous speech, formants (F1 and F2), pitch perturbation quotient (PPQ), relative average perturbation (RAP), noise-to-harmonic ratio (NHR), and intensity. Participants also completed a selfevaluation of communicative experiences using a five-point scale from the Transsexual Voice Questionnaire, Male-to-Female (TVQMtF). Speech samples were collected pre- and post-training to establish statistical and clinical significance.

Results: Statistically and clinically significant gains were found between pre- and post-training in the areas of F0 in vowel prolongation, and SFF during spontaneous speech. There were generally medium effect sizes for F1 and F2, PPQ, RAP, NHR, and intensity. These analyses were limited by sample size. Results suggest that F0 of vowel prolongation, SFF, SFF variation, formant modification, and intensity are suitable targets for evidence-based practices for transgender women.
\end{abstract}




\section{ACKNOWLEDGMENTS}

I would like to thank everyone who provided support and guidance to complete this thesis. All mistakes are mine alone. I would first like to thank my advisor and academic supervisor Dr. Leslie Mahler. Her patience, thoughtfulness, and confidence in my abilities helped me to persevere throughout this process. Thank you to Kimberly Dahl, your hard work has provided an opportunity for which I will be eternally grateful. Thank you to Madison Turner, for all of her hard work and patience, I hope this experience was of value. Thanks are due to my thesis committee, from whom I have learned to better myself personally and professionally. I would also like to thank my husband, whose unwavering patience and support has allowed me to change careers, and work toward my personal and professional goals.

Lastly, I am most thankful of all of the participants who found time to take part in this research. The opportunity to meet these individuals is something I will cherish the most from this experience. 


\section{DEDICATION}

For my transgender friends and family, and all members of the LGBTQ+ community. 


\section{TABLE OF CONTENTS}

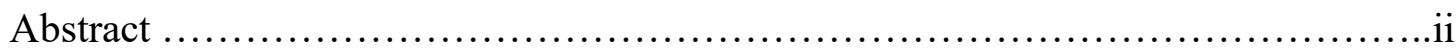

Acknowledgments ......................................................................ii

Dedication .........................................................................

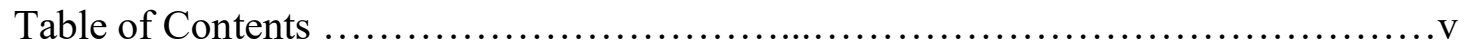

List of Tables ..............................................................

List of Figures .............................................................

Chapter 1: Introduction ......................................................

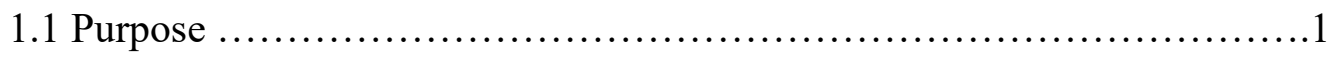

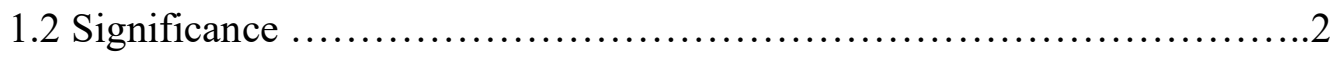

Chapter 2: Background ........................................................

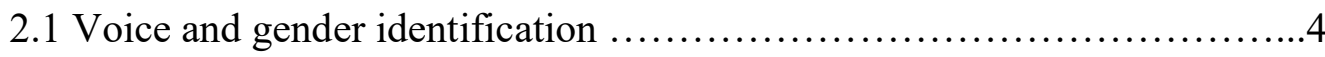

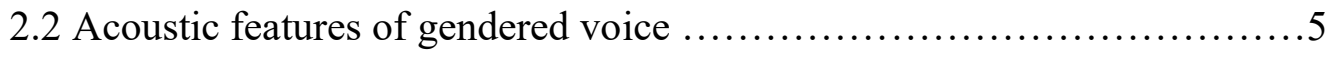

2.3 Self-perception of the feminine voice ...............................6

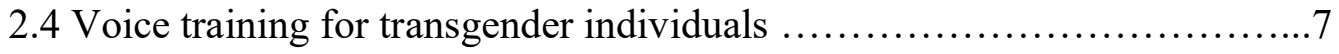

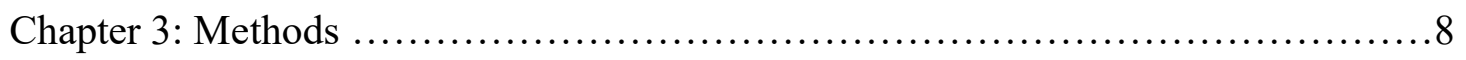

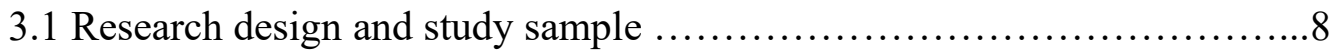

3.2 Acoustic variables obtained from voice and speech samples pre- and post-

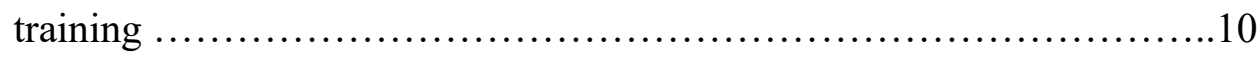

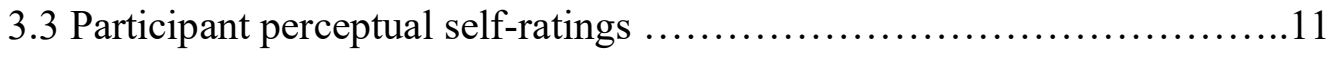

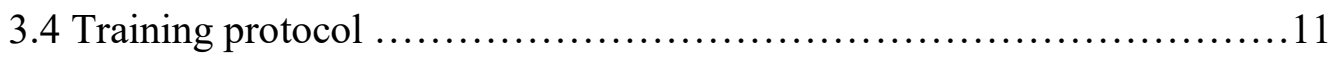

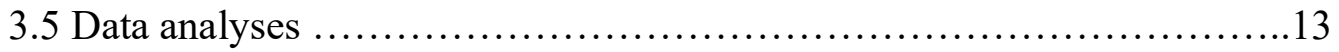


Chapter 4: Results 16

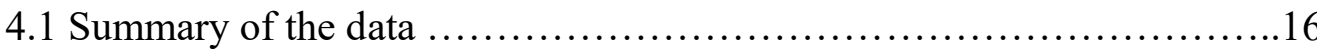

4.2 Research aim 1: Measure acoustic variables of voice and speech related to gender identity immediately before and immediately after six weeks of a transgender voice training protocol ................................. 35

4.3 Research aim 2: Determine whether quantitative changes in acoustic features related to gender identity relate to perceptual measures of voice and speech

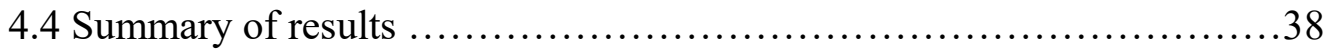

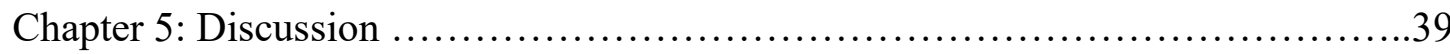

5.1 Aim 1 Hypothesis: Acoustic variables will more closely align with cisgender women post-training. .....................................

5.2 Aim 2 Hypothesis: As acoustic variables more closely align with cisgender women post training, voice satisfaction will improve

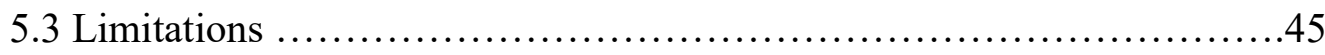

Chapter 6: Conclusions ..............................................48

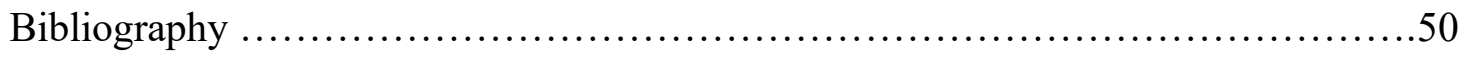




\section{LIST OF TABLES}

Table 3.1 List of Participants and Demographic Information ......................9

Table 3.2 List of Dependent Variables .................................... 14

Table 4.1 F0 of Vowel Prolongation by Participant ............................ 18

Table 4.2 Speaking Fundamental Frequency by Participant $\ldots \ldots \ldots \ldots \ldots \ldots \ldots \ldots \ldots . .21$

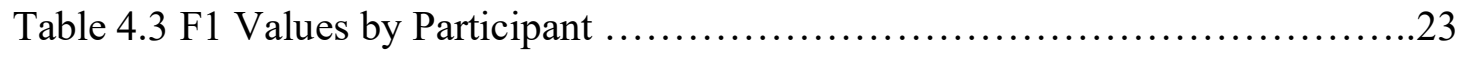

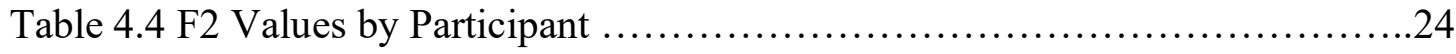

Table 4.5 PPQ Values by Participant ..........................................27

Table 4.6 RAP Values by Participant ......................................28

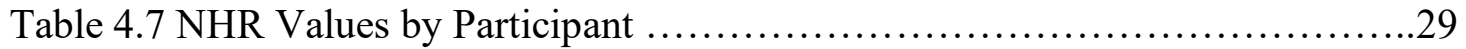

Table 4.8 Intensity of Vowel Prolongation by Participant ........................... 31

Table 4.9 Intensity of Spontaneous Speech Tasks by Participant ...................32

Table 4.10 TVQMtF Score Sums by Category, Pre- and Post- Training 34

Table 4.11 Mean TVQmtF Differences by

Category $\ldots \ldots \ldots \ldots \ldots \ldots \ldots \ldots \ldots \ldots \ldots . \ldots \ldots \ldots$ 


\section{LIST OF FIGURES}

Figure 4.1 Mean SFF by Participant Derived from Picture Description Samples ......20

Figure 4.2 Mean SFF by Participant Derived from Monologue Samples ............20 


\section{Chapter 1}

\section{INTRODUCTION}

Voice training for transgender (trans) individuals is an area in which the field of speech-language pathology knows little. As a result, the trans community has received little consideration in the area of speech and communication research. The World Professional Association for Transgender Health (WPATH) did not establish standards of care for speech or voice until its seventh edition published in 2012 (Standards of care for the health of transsexual, transgender, and gendernonconforming people, 2012). Society has evolved to become a culturally diverse community. Speech language pathologists and other scientists are now charged with developing knowledge and understanding of methods and services to better provide support and evidence-based treatments to the LGBTQ+ community.

Selecting appropriate speech and voice targets for trans women is vital to establishing an effective protocol for training. Presentation of a gender congruent voice as a desired outcome for training is often a goal for trans women seeking voice training. Objective data and perceptual data are needed to clearly comprehend how specific approaches to training affect speech and voice.

\subsection{Purpose}

The purpose of this study is to contribute to the knowledge base and provide data to evaluate the efficacy of a specific speech and voice training protocol. It examines 
the impact that this protocol has on acoustic features of 10 trans women pre- and posttraining. The specific aims of the study are:

1. Measure acoustic and perceptual variables of voice and speech related to gender identity immediately before and immediately after six weeks of a transgender voice training protocol.

a. Hypothesis: Changes in acoustic variables will result in speech and voice characteristics that more closely align with cisgender women post-training.

2. Determine whether quantitative changes in acoustic features related to gender identity correlate with self-perceptual measures of voice and speech.

a. Hypothesis: As changes in acoustic variables more closely align with cis-gender women post-training, voice satisfaction will improve.

\subsection{Significance}

This study contributes to the literature in numerous ways. First, it involves analyses of speech and voice measures, some of which have received little scrutiny in past studies of the trans population. Second, it compares pre- and post-training data using a self-perceptual rating of voice. Third, it provides data regarding vocal characteristics in sustained vowel phonation and connected speech during spontaneous productions in salient speech contexts. This study provides insight into appropriate goals and objectives that are likely to promote generalization outside of training. 
Lastly, this research identifies differences in pre- and post-training speech and voice variables. Determining the statistical and clinical significance of these differences are essential to providing information for speech language pathologists, and to establish targets that are likely to be most effective in helping trans individuals to achieve their goals of gender-congruent communication. 


\section{Chapter 2}

\section{BACKGROUND}

Previous research of training programs for transgender women is lacking in the current literature. Research that is available does not consistently include a clearly described and specific training protocol. This chapter provides a description of the previous literature and establishes the need to clarify and further establish the need for additional research on voice training for trans individuals.

\subsection{Voice and gender identification}

Several vocal characteristics have been studied in previous research studies to identify whether they make significant differences in gender identification. Gelfer and Schofield's (2000) analysis between vowel formant frequency, gender perception, and ratings of femininity indicated minimal clinical significance between acoustic features and femininity-masculinity rating scale. However, results did demonstrate significant differences between speaking fundamental frequency (SFF) and upper limit of SFF, as subjects with high SFF (mean $187 \mathrm{~Hz})$ and SFF limit $(165-209 \mathrm{~Hz})$ were more often perceived as female. The study also noted that subjects' progress varied as a result of differences in the stage of transition (e.g., initial, medial, or final stages of transition).

Research findings by Gorham-Rowan and Morris (2006) revealed that ratings of trans women by unfamiliar listeners were most likely to be identified as female when speaking with an SFF of $160 \mathrm{~Hz}$ and greater. Increasing pitch along with other voice and speech modification strategies, such as modifying loudness (Hancock \& Garabedian, 2012), modifying (Quinn \& Swain, 2018), and following healthy vocal 
hygiene practices have been shown to increase the likelihood of gender congruent speech perception (Dahl and Mahler, 2019).

\subsection{Acoustic features of gendered voice}

Cisgender individuals, those who identify with the gender they were born with, display specific vocal characteristics which coincide with societal norms. These features provide acoustic information to listeners and allow them to perceive a voice as masculine or feminine. Cisgender women's SFF is generally produced at an average of $250 \mathrm{~Hz}$ (Kumar, Banumathy, Sharma \& Panda, 2019).

Post-pubescent males tend to have much longer and thicker vocal folds and a larger vocal tract (Kawitzky and McAllister, 2020) when compared to women. Trans women who speak at an F0 lower than $160 \mathrm{~Hz}$ have a greater chance of being misgendered when physical cues (i.e., physical presentation) are not available (i.e., during telephone calls) (Kawitzky and McAllister, 2020).

Podesva and Callier (2015) described voice quality as "the extragrammatical suprasegmental properties of speech resulting from the configuration of the vocal apparatus." Resonance plays a key role in voice. The glottal wave, created by vibration of the vocal folds, travels through the vocal tract. The vocal tract modifies the signal heard by listeners. This altered soundwave is what listeners perceive as the speaker's voice. Feminine voices tend to contain higher formants, which provide auditory cues to listeners and allow them to perceive the gender of the speaker as female (King, Brown, and McCrea, 2012). Formants vary by speaker, specifically in vowel production. They rely on length and shape of the vocal tract and tongue 
placement. Formants are an important factor in the perception of gender (Kawitzky and McAllister, 2020).

\subsection{Self-perception of the feminine voice}

Self-perception of vocal femininity is a vital factor in self-acceptance of an individual's identity (Lara and Peterson, 2018). Perceptual tools used to establish a self-rating of vocal femininity include the Transsexual Voice Questionnaire - Male to Female (TVQMtF) and the Transgender Self-Evaluation Questionnaire (TSEQ). Both questionnaires contain similar questions concerning social interaction, emotions, gender and identity, speech production effort, physical aspects, and pitch (Davies and Johnston, 2015). Dahl and Mahler (2019) found a moderately positive correlation between self-ratings and maximum SFF, utilizing the TVQMtF. Casado, RodriguezParra, and Adrian (2016) used the TSEQ in their research. They noted a moderately significant correlation between SFF and the TSEQ score.

Few studies have sought to establish relationships between acoustic features and self-perception beyond SFF. Hancock and Garabedian (2013) studied 25 cases to correlate self-perception with SFF and semitone ranges, which produced conflicting results. Participants who presented an SFF within a masculine range reported high satisfaction with their voice. This finding suggests that even those who did not achieve their SFF goals developed self-confidence by using a higher pitch. Therefore, a study of training outcomes for trans women should include a combination of quantitative assessment of acoustic vocal features and a qualitative assessment of self-perception to understand participant's self-perception of their communication and communication 
experiences. This study will use acoustic measures and measures of self-perception to gain a comprehensive understanding of the outcomes of speech and voice training.

\subsection{Voice training for transgender individuals}

Voice congruency is an important component in the transition of trans individuals, but little is known about specific training strategies that are most effective. A lack of research in voice modification training techniques for trans individuals leads to the question; What training techniques are most effective for trans women to achieve their communication goals? Specifying a voice training protocol for trans individuals is a crucial area for study because there is a lack of research investigating the most effective evidence-based practices for this underserved population. Training protocols should target the most salient features of gendered communication including acoustic speech variables and perceptions of the speaker's voice.

This study contributes to the literature by measuring changes in vocal characteristics of trans women following a specific training protocol. Analyses will permit the comparison of changes in acoustic variables and self-reported satisfaction in the areas of voice and communication. The study will allow examination of the effect of training and identify how it affects acoustic variables. It will assist in the development of a specific protocol for a speech-language pathologist to provide best practices and appropriate treatment goals for trans women. 


\section{Chapter 3}

\section{METHODS}

\subsection{Research design and study sample}

This study is a multiple single-subject experimental research design that includes analysis of pre- and post-training data for 10 trans women. Eligibility criteria for

participants were: (1) identification as a trans woman, (2) aged 18-78 years, and (3) no history of laryngeal trauma or surgery. Vocal changes related to aging and surgery or trauma are likely to affect acoustic and self-perception measures of voice, resulting in confounding study results. Consequently, these confounding variables resulted in a participant's exclusion from the study.

The participants ranged in age from 25 to 60 and the amount of time spent in transition varied. Table 3.1 provides a summary of descriptive information about the participants. 


\begin{tabular}{|c|c|c|c|c|}
\hline \multirow[b]{2}{*}{ Participant Code } & \multicolumn{3}{|c|}{ Previous } & \multirow[b]{2}{*}{ Medications } \\
\hline & Age & $\begin{array}{l}\text { Feminization } \\
\text { Training? }\end{array}$ & $\begin{array}{l}\text { Years in } \\
\text { Transition }\end{array}$ & \\
\hline TV202 & 35 & Yes & $<1$ & Spironolactone, estradiol \\
\hline TV206 & 44 & Yes & 1 & $\begin{array}{c}\text { Spironolactone, estradiol, } \\
\text { finasteride }\end{array}$ \\
\hline TV208 & 39 & Yes & 2 & $\begin{array}{c}\text { Spironolactone, estradiol, } \\
\text { finasteride, } \\
\text { medroxyprogesterone }\end{array}$ \\
\hline TV209 & 29 & Yes & - & $\begin{array}{l}\text { Spironolactone, estradiol, } \\
\text { finasteride }\end{array}$ \\
\hline TV210 & 29 & No & $<1$ & Spironolactone, estradiol \\
\hline TV211 & 31 & No & $<1$ & Spironolactone, estradiol \\
\hline TV301 & 60 & No & - & $\begin{array}{c}\text { Spironolactone, estradiol, } \\
\text { finasteride }\end{array}$ \\
\hline TV302 & 20 & No & $<1$ & Estradiol, finasteride \\
\hline TV303 & 29 & No & $<1$ & None \\
\hline TV304 & 26 & No & $<1$ & None \\
\hline
\end{tabular}

Recruitment of trans participants took place by circulating IRB-approved brochures to the gender and sexuality clinics at Rhode Island Hospital and Thundermist clinics. Notices were also distributed through LGBTQ+ organizations including: The University of Rhode Island (URI) Gender and Sexuality Center, Rhode Island Pride, TGI Network of Rhode Island, and Sojourner House. Previous URI Transgender Voice Clinic participants were invited to partake in the study. 


\subsection{Acoustic variables obtained from voice and speech samples pre- and post-training}

Evaluations of voice were completed during two 30 -minute sessions at the University of Rhode Island's (URI) Speech and Hearing Center in Kingston, RI. Initial appointments consisted of an interview to collect demographic information (i.e., medications, previous voice modification practices, and overall transition).

Participants engaged in the same speech and voice tasks in a sound booth during each evaluation. The speech and voice tasks included:

1. Vowel /a, i, $\mathrm{u} /$ prolongations (3 repetitions)

1.1. Dependent variables: F0, variation, F1 \& F2, intensity, pitch perturbation quotient (PPQ), relative average perturbation (RAP), noise-to-harmonic ratio (NHR)

2. Short sentence reading containing the vowels mentioned above (3 repetitions)

2.1. Dependent variables: F0, variation, intensity

3. Reading of a section of The Rainbow Passage

3.1. Dependent variables: F0, variation, intensity

4. Illustrated scene description (i.e., the cookie theft picture of the Boston Diagnostic Aphasia Examination)

4.1. Dependent variables: F0, variation, intensity

5. Monologue in reply to a prompt (i.e., Tell me your proudest moment)

5.1. Dependent variables: F0, variation, intensity

Data were collected during two evaluations to account for vocal inconsistencies resulting from everyday activities such as sleep, health, hydration, diet, 
irritation due to irritant exposure, etc. A Countryman omnidirectional, head-mounted microphone was utilized at a standard distance of $8 \mathrm{~cm}$ from the center of each participants' lips. Samples were recorded with GoldWave v6.21 software in a sound isolation booth at the URI Speech and Hearing Center, using a Universal Audio 4710D preamplifier and an RME Fireface UC audio interface.

\subsection{Participant Perceptual Self-ratings}

Each participant completed the Transsexual Voice Questionnaire - Male to Female (TVQMtF) during the initial evaluation and post-training evaluation. The TVQMtF results were used to address participants' self-perception of their voice by utilizing a five-point Likert Scale to establish a rating of communicative experiences. The same scale was presented during an exit interview for each participant. Ratings collected pre- and post-training were analyzed and evaluated with acoustic variables. This was completed to determine if changes in SFF has an influence on self-perception of voice and speech. The TVQMtF questions were grouped into six categories related to an individual's speech and communication according to Davies and Johnston (2015). These categories include; (a) social interaction, (b) emotional implications on communication, (c) gender and identity, physical aspects of speech, and (d) pitch. Davies and Johnston (2015) also studied the validity of the TVQMtF and determined that 24 percent of the items on the questionnaire did not pertain to voice but were considered appropriate with regard to the experiences of trans women. Reliability of the TVQMtF was found to have internal consistency, and excellent test-retest reliability (Dacakis et al., 2013). 


\subsection{Training Protocol}

Voice training took place three times per week, for 50 minutes per session, for six weeks. Sessions consisted of two individual sessions and one group session each week. Training was provided by clinicians instructed in the area of voice. Based on the pre-training evaluation and data collection, a target pitch was established for each participant. Each session began with exercises for participants to prepare their vocal folds for healthy voice production of an increased pitch. Vocal warmups consisted of semi-occluded vocal tract exercises, sustained /i/, and pitch glides.

Participants produced phonation through a straw during the semi-occluded vocal tract exercises, to reduce vocal fold tension while ranging from highest to lowest comfortable pitch for 5-10 seconds. During sustained /i/ exercises, participants were instructed to produce and sustain the vowel /i/ at their target pitch for 5 to 10 seconds. The final exercise, pitch glides, required participants to produce the /i/ sound again and gradually increase pitch as high as comfortably possible. The exercise was then repeated while decreasing the pitch as low as comfortably possible.

Vocal strategies from non-speaking tasks were integrated into functional speech, using phrases and sentences determined by the participants based on their daily communication. Participants read their functional phrases using a higher SFF and greater variation of pitch. These sentences consisted of phrases which the individual stated on a regular basis to promote generalization of the participant's targeted SFF. The remainder of the sessions consisted of various tasks and activities to encourage spontaneous speech productions utilizing the participant's targeted SFF. 
Additional tasks targeted other verbal and nonverbal characteristics of communication (i.e., intonation, light articulation, gestures, and body language). At the conclusion of each session, clinicians discussed proper vocal hygiene practices (i.e., proper hydration, no shouting, vocal rest, etc.). Vocal hygiene education allowed participants to maintain adequate vocal health during the six-week training period. Clinicians also provided carry-over assignments, which involved communicating with a loved one or making a telephone call using their targeted SFF.

Group sessions involved three to six participants. Each group session began with participants taking turns practicing their targeted pitch while phonating /i/ for five seconds to calibrate to the desired SFF. The remainder of the session involved tasks which required participants to ask questions, describe items, express opinions, and contribute to conversations. Clinicians monitored the SFF of each participant while they spoke and provided verbal and/or gestural cues as needed to adjust participants' pitch. The main goal of group sessions was to maintain target SFF during spontaneous speech in a supported environment that more closely approximated functional communications situations. While individual sessions directly focused on target SFF, intonation variation, and the individual needs of each participant.

\subsection{Data Analyses}

F0, pitch perturbation quotient (PPQ), relative average perturbation (RAP), and noise-to-harmonic ratio (NHR) were computed for vowel prolongation samples from each evaluation. F0 was analyzed utilizing the Multi-Dimensional Voice Program (MDVP), which provided a mean F0 and standard deviation as a variation measure. 
PPQ analyzes the relative variability of frequency from point to point within a speech sample, with a smoothing factor of 5 points. RAP analyzes the relative variability of frequency from point to point within a speech sample, with a smoothing factor of 3 points. These measures are commonly referred to as jitter and provide information about vibratory patterns of the vocal folds. NHR defines the average ratio of inharmonic spectral energy within a high frequency range $(1500-4500 \mathrm{~Hz})$ to the harmonic spectral energy within the SFF range $(70-4500 \mathrm{~Hz})$. This defines the amount of noise present within a signal (Dejonckere et al., 2007).

Table 3.2 lists the dependent variables and the analysis tools used along with measures that were analyzed, the task from which the values were derived, and software utilized. To ensure inter-rater reliability, 20 percent of the samples were analyzed by a speech-language pathology graduate student unaffiliated with the study.

\begin{tabular}{ccc} 
Table 3.2 List of dependent variables & & \\
\hline Dependent Variables & Task & Software \\
\hline SFF $(\mathrm{Hz})$ & Spontaneous speech samples & PRAAT \\
SFF Variation $(\mathrm{Hz})$ & Spontaneous speech samples & PRAAT \\
F1 \& F2 (Hz) & Vowel Prolongation & PRAAT \\
PPQ (\%) & Vowel prolongation & Computerized speech lab \\
RAP (\%) & Vowel prolongation & Computerized speech lab \\
NHR (dB SPL) & Vowel prolongation & Computerized speech lab \\
Self-perception rating & TVQMtF & Excel \\
\hline
\end{tabular}

Differences between pre- and post-training data were determined via dependent, two-tailed t-tests. The above measures were also correlated with the following variables: (1) TVQMtF results pre-voice training and; (2) TVQMtF results post-voice training. A non-directional hypothesis is reasonable for research on voice and gender, affirming the use of a two-tailed test. Voices of adult females are characterized by increased pitch (Oates \& Dacakis, 2015), higher vowel formants 
(Gelfer \& Schofield, 2000), and varying intonation (Dahl \& Mahler, 2019) when compared to adult males. 


\section{Chapter 4}

\section{RESULTS}

This chapter presents the results for each of the variables of interest in this study. The data collected are summarized in the tables below. These data include: (1) acoustic measures collected from speech samples of trans women, and (2) self-ratings of voice femininity. The results related to each research aim of this study are also presented.

\subsection{Summary of the data}

This study analyzed acoustic variables of voice and speech and self-perceptual ratings of voice and communication for all participants. These data are reported by variable for each participant. The researchers used $\alpha=0.05$ as the level for statistical significance. Effect size (r) and Cohen's correlation coefficient (d) were also calculated using the University of Colorado, Colorado Springs' effect size calculator, presented by Becker (2000) to evaluate clinical significance of changes following training. Effect sizes were assessed using Cohen's (d): a value of $d<0.20$ was considered little effect, 0.20 to 0.49 small, 0.50 to 0.79 medium effect, and $>0.80$ as large effect.

Table 4.1 provides a summary of Fundamental Frequency (F0) during vowel prolongations of $/ \mathrm{a}, \mathrm{i}$, and $\mathrm{u} /$ for each participant, pre- and post-training. Frequencies of the post-training evaluations were consistently higher than pre-training evaluations for all vowels and across all participants. Pre-training evaluations revealed that four 
out of ten participants' average F0 was below the expected frequency range for gender-ambiguous individuals (145 - $165 \mathrm{~Hz}$ ) (Dahl and Mahler, 2019).

P-values for F0 of vowel prolongation revealed statistically significant increases $(\mathrm{p}=<0.01$ to 0.02$)$ across all participants. Effect size analyses revealed a large effect size between F0 of vowel prolongation pre- and post-training for all vowels $(\mathrm{r}=0.47$ to 1.00$)$. F0 of vowel prolongation was higher post-training than pretraining. The highest recorded frequencies were not associated with any single vowel. 
Table 4.1 Fo of Vowel Prolongation by Participant

\begin{tabular}{|c|c|c|c|c|c|c|c|c|}
\hline Part. ID & & $\begin{array}{c}\text { Pre } \\
\text { Mean }\end{array}$ & $\mathrm{SD}$ & $\begin{array}{c}\text { Post } \\
\text { Mean }\end{array}$ & SD & p-value & $\begin{array}{c}\text { Cohen's } \\
\text { d }\end{array}$ & $\begin{array}{c}\text { Effect size } \\
\mathbf{r}\end{array}$ \\
\hline \multirow{3}{*}{ TV202 } & $/ \mathbf{a} /$ & - & - & 236.00 & 3.95 & - & - & - \\
\hline & $/ \mathbf{i} /$ & 170.30 & - & 330.72 & 12.57 & - & 18.05 & 0.99 \\
\hline & $/ \mathbf{u} /$ & 166.75 & 9.33 & 255.31 & 8.71 & $<0.01$ & 9.82 & 0.98 \\
\hline \multirow{3}{*}{ TV206 } & $/ \mathbf{a} /$ & 127.11 & 14.19 & 193.87 & 4.83 & $<0.01$ & 6.30 & 0.95 \\
\hline & $/ \mathbf{i} /$ & 134.02 & 15.26 & 205.89 & 1.85 & $<0.01$ & 6.61 & 0.96 \\
\hline & $/ \mathbf{u} /$ & 138.06 & 14.35 & 208.81 & 1.37 & $<0.01$ & 6.94 & 0.96 \\
\hline \multirow{3}{*}{ TV208 } & $/ \mathbf{a} /$ & 167.37 & 8.09 & 227.55 & 33.47 & $<0.01$ & 2.47 & 0.78 \\
\hline & $/ \mathbf{i} /$ & 165.78 & 3.75 & 307.62 & 12.75 & $<0.01$ & 15.09 & 0.99 \\
\hline & $/ \mathbf{u} /$ & 170.81 & 15.23 & 316.93 & 13.16 & $<0.01$ & 10.27 & 0.98 \\
\hline \multirow{3}{*}{ TV209 } & $/ \mathbf{a} /$ & 154.65 & 2.47 & 217.06 & 1.75 & $<0.01$ & 29.15 & 1.00 \\
\hline & $/ \mathbf{i} /$ & 177.68 & 13.66 & 269.89 & 1.36 & 0.01 & 9.50 & 0.98 \\
\hline & $/ \mathbf{u} /$ & 167.11 & 5.77 & 254.40 & 0.53 & $<0.01$ & 21.30 & 1.00 \\
\hline \multirow{3}{*}{ TV210 } & $/ \mathbf{a} /$ & 183.25 & 11.30 & 202.80 & 23.47 & 0.01 & 1.06 & 0.47 \\
\hline & $/ \mathbf{i} /$ & 186.02 & 12.66 & 209.91 & 20.75 & $<0.01$ & 1.39 & 0.57 \\
\hline & $/ \mathbf{u} /$ & 185.20 & 13.37 & 213.47 & 19.36 & $<0.01$ & 1.70 & 0.65 \\
\hline \multirow{3}{*}{ TV211 } & $/ \mathbf{a} /$ & 126.07 & 1.80 & 210.43 & 14.29 & $<0.01$ & 8.28 & 0.97 \\
\hline & $/ \mathbf{i} /$ & 130.88 & 1.89 & 212.28 & 12.56 & $<0.01$ & 9.06 & 0.57 \\
\hline & $/ \mathbf{u} /$ & 136.29 & 2.22 & 216.20 & 13.15 & $<0.01$ & 8.47 & 0.97 \\
\hline \multirow{3}{*}{ TV301 } & $/ \mathbf{a} /$ & 102.54 & 4.68 & 177.54 & 6.41 & $<0.01$ & 13.36 & 0.99 \\
\hline & $/ \mathbf{i} /$ & 103.36 & 3.71 & 198.35 & 2.44 & $<0.01$ & 30.27 & 1.00 \\
\hline & $/ \mathbf{u} /$ & 102.63 & 3.37 & 204.62 & 2.02 & $<0.01$ & 36.73 & 1.00 \\
\hline \multirow{3}{*}{ TV302 } & $/ \mathbf{a} /$ & 155.06 & 2.02 & 283.14 & 2.28 & $<0.01$ & 59.50 & 1.00 \\
\hline & $/ \mathbf{i} /$ & 176.73 & 3.10 & 274.46 & 19.90 & $<0.01$ & 6.86 & 0.96 \\
\hline & $/ \mathbf{u} /$ & 181.68 & 3.06 & 287.74 & 4.21 & $<0.01$ & 28.82 & 1.00 \\
\hline \multirow{3}{*}{ TV303 } & $/ \mathbf{a} /$ & 171.46 & 1.92 & 187.57 & 11.04 & 0.02 & 2.03 & 0.71 \\
\hline & $/ \mathbf{i} /$ & 176.69 & 1.10 & 192.30 & 8.46 & 0.01 & 2.59 & 0.79 \\
\hline & $/ \mathbf{u} /$ & 177.76 & 0.98 & 195.87 & 11.42 & 0.02 & 2.23 & 0.75 \\
\hline \multirow{3}{*}{ TV304 } & $/ \mathbf{a} /$ & 106.04 & 1.32 & 196.28 & 16.32 & $<0.01$ & 7.80 & 0.97 \\
\hline & $/ \mathbf{i} /$ & 113.14 & 1.64 & 222.48 & 9.72 & $<0.01$ & 15.68 & 0.99 \\
\hline & $/ \mathbf{u} /$ & 114.10 & 1.01 & 230.91 & 2.63 & $<0.01$ & $\mathbf{5 8 . 5 7}$ & 1.00 \\
\hline
\end{tabular}

$\mathrm{SD}=$ Standard Deviation 
Table 4.2 summarizes the average speaking fundamental frequency (SFF) of spontaneous speech samples during picture description and monologue tasks. SFF was clinically significantly higher during post-training evaluations than during pre-training evaluations across all participants for picture description and for 9 out of 10 participants during a monologue. SFF decreased from 162.24 to 152.33 for participant TV202.

The mean SFF of spontaneous speech samples revealed a trend of increased SFF immediately post-training. The picture description task showed more dramatic changes in the mean SFF across all samples between pre- and post-training values (pre-training mean $=139.08 \mathrm{~Hz}, \mathrm{SD}=25.88$; Post-training mean $=172.45 \mathrm{~Hz}, \mathrm{SD}=$ 17.92). The difference in means was $+33.37 \mathrm{~Hz}$. Though there was a smaller difference between the pre- and post-training means calculated from the monologue samples, the difference in means presented a substantial difference (pre-training mean $=142.00 \mathrm{~Hz}, \mathrm{SD}=22.62$; post-training mean $=158.73 \mathrm{~Hz}, \mathrm{SD}=17.57)$. It should be noted that one sample presented lower SFF upon analysis of the post-training sample $($ mean $\mathrm{SFF}=152.33 \mathrm{~Hz}, \mathrm{SD}=52.49)$ than the pre-training sample (Mean $\mathrm{SFF}=$ 162.24 Hz, SD = 49.49). 
Figure 4.1 Mean SFF by Participant Derived from Picture Description Samples

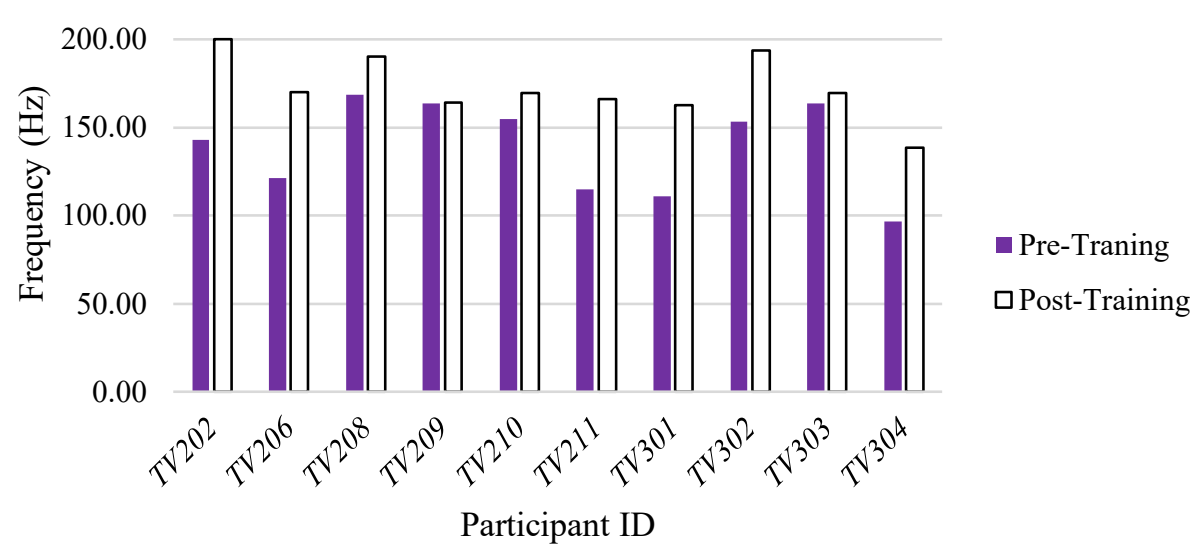

Figure 4.2 Mean SFF by Participant Derived from Monologue Samples

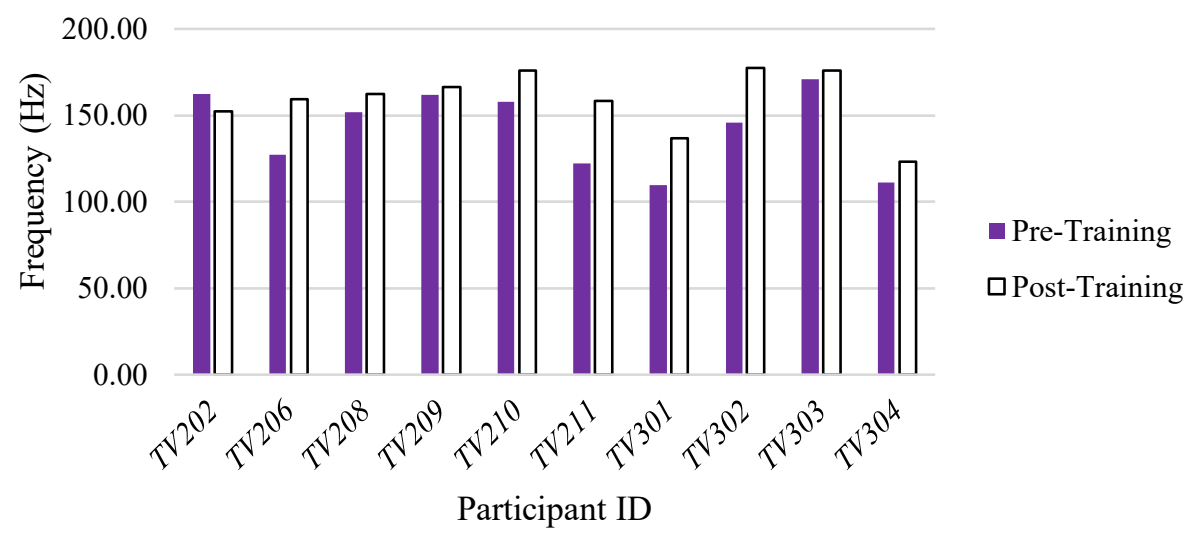

There were statistically significantly increases in SFF for 9 out of 10 participants during the picture description task $(\mathrm{p}=<0.01$ to 0.05$)$, and for 7 out of 10 participants for the monologue task ( $\mathrm{p}=<0.01$ to 0.04 ). Effect size was calculated for picture description and monologue using Cohen's (d) and Effect size (r). The effect size was large for 6 out of 10 participants for picture description $(r=0.30$ to 0.67$)$. The remaining four samples had a low-to-medium effect size $(r=0.11$ to 0.23$)$. Effect size was large for only 2 of 10 participants for monologue samples ( $r=0.40$ to 0.46$)$. 
Three participants had a medium effect size range $(r=0.16$ to 0.35$)$, and the remaining

5 had a small effect size $(r=0.09$ to 0.15$)$.

Table 4.2 Speaking Fundamental Frequency by Participant

\begin{tabular}{|c|c|c|c|c|c|c|c|c|}
\hline $\begin{array}{l}\text { Part. } \\
\text { ID }\end{array}$ & & Pre Mean & SD & $\begin{array}{c}\text { Post } \\
\text { Mean }\end{array}$ & SD & p-value & $\begin{array}{c}\text { Cohen's } \\
\text { d }\end{array}$ & $\begin{array}{l}\text { Effect } \\
\text { size } r\end{array}$ \\
\hline \multirow{2}{*}{ TV202 } & Pic. Desc. & 142.87 & 71.70 & 199.95 & 61.09 & $<0.01$ & 0.86 & 0.39 \\
\hline & Monologue & 162.24 & 49.49 & 152.33 & 52.49 & 0.02 & 0.19 & 0.10 \\
\hline \multirow{2}{*}{ TV206 } & Pic. Desc. & 121.12 & 38.30 & 170.06 & 52.65 & $<0.01$ & 1.06 & 0.47 \\
\hline & Monologue & 127.26 & 35.87 & 159.15 & 71.39 & 0.02 & 0.56 & 0.27 \\
\hline \multirow{2}{*}{ TV208 } & Pic. Desc. & 168.70 & 49.78 & 190.26 & 40.95 & 0.05 & 0.47 & 0.23 \\
\hline & Monologue & 151.71 & 26.64 & 162.27 & 38.01 & 0.23 & 0.32 & 0.16 \\
\hline \multirow[b]{2}{*}{ TV209 } & Pic. Desc. & 163.67 & 79.42 & 164.16 & 38.85 & 0.13 & 0.47 & 0.23 \\
\hline & Monologue & 162.03 & 43.16 & 166.42 & 41.67 & 0.04 & 0.32 & 0.16 \\
\hline \multirow{2}{*}{ TV210 } & Pic. Desc. & 154.62 & 58.01 & 169.51 & 53.28 & $<0.01$ & 0.27 & 0.13 \\
\hline & Monologue & 157.63 & 60.64 & 175.85 & 55.40 & $<0.01$ & 0.31 & 0.15 \\
\hline \multirow{2}{*}{ TV211 } & Pic. Desc. & 115.08 & 37.10 & 165.93 & 34.81 & $<0.01$ & 1.41 & 0.58 \\
\hline & Monologue & 121.94 & 37.33 & 158.16 & 32.41 & $<0.01$ & 1.04 & 0.46 \\
\hline \multirow[b]{2}{*}{ TV301 } & Pic. Desc. & 110.83 & 71.70 & 199.95 & 61.09 & $<0.01$ & 0.86 & 0.39 \\
\hline & Monologue & 109.46 & 17.20 & 136.68 & 48.20 & $<0.01$ & 0.75 & 0.35 \\
\hline \multirow[b]{2}{*}{ TV302 } & Pic. Desc. & 153.31 & 40.23 & 193.63 & 54.75 & $<0.01$ & 0.39 & 0.00 \\
\hline & Monologue & 145.76 & 26.70 & 177.41 & 43.23 & $<0.01$ & 0.40 & 0.00 \\
\hline \multirow{2}{*}{ TV303 } & Pic. Desc. & 163.73 & 25.24 & 169.75 & 28.23 & $<0.01$ & 0.23 & 0.11 \\
\hline & Monologue & 170.66 & 20.02 & 175.68 & 32.81 & 0.11 & 0.19 & 0.09 \\
\hline \multirow{2}{*}{ TV304 } & Pic. Desc. & 96.84 & 20.59 & 138.73 & 25.29 & $<0.01$ & 1.82 & 0.67 \\
\hline & Monologue & 111.33 & 29.57 & 123.39 & 25.49 & 0.62 & 0.44 & 0.21 \\
\hline
\end{tabular}

Tables 4.3 and 4.4 summarize the frequencies of vowel formants (F1 and F2)

for $/ \mathrm{a}, \mathrm{i}$, and $\mathrm{u} /$ produced by each participant during sustained vowel phonation. There were inconsistencies in formant production across participants. Similar to SFF, the means for these values also fell between the means of gender ambiguous individuals (145 - $165 \mathrm{~Hz})$ (Dahl and Mahler, 2019).

Formant frequency changes for the participants of this study were inconsistent throughout the analyses. With regard to F1, $p$-values varied across vowels $(p=<0.01$ 
to 0.72 ). Five participants made statistically significantly increases in frequency of F1 during sustained vowel phonation of $/ \mathrm{a} /(\mathrm{p} \leq 0.05)$, and 6 participants statistically significantly increased F1 frequency for each of the vowels /i/ and /u/. No individual participant demonstrated improved F1 values across all three vowels. Two participants statistically significantly increased the frequency of F2 for the vowel /a/, seven participants statistically significantly increased the frequency of F2 for the vowel /i/, and four participants for the vowel /u/. No single participant demonstrated improved F2 values across all vowels. Approximately half of the participants' productions of F1 and F2 had medium-to-large effect sizes for sustained vowel phonation $(r=0.39$ to 0.99). Seven participants clinically significantly increased their frequency of F1 during the production the vowel /a/ $(\mathrm{r}=0.42$ to 0.93$)$ and seven participants clinically significantly increased F1 frequency for each the vowels $/ \mathrm{i} /$ and $/ \mathrm{u} /(\mathrm{r}=0.90$ to 0.97$)$. Seven participants made clinically significantly increases in frequency of F2 for the vowel /a/, eight participants clinically significantly increased the frequency of F2 for the vowel /i/, and six participants for the vowel /u/. No consistent patterns within participants or vowels were detected within formant frequency analyses. 
Table 4.3 F1 Values by Participant

\begin{tabular}{|c|c|c|c|c|c|c|c|c|}
\hline Part. ID & & $\begin{array}{c}\text { Pre } \\
\text { Mean }\end{array}$ & SD & $\begin{array}{c}\text { Post } \\
\text { Mean }\end{array}$ & SD & p-value & $\begin{array}{c}\text { Cohen's } \\
\text { d }\end{array}$ & $\begin{array}{c}\text { Effect size } \\
\mathbf{r} \\
\end{array}$ \\
\hline \multirow{3}{*}{ TV202 } & $/ \mathbf{a} /$ & - & - & 655.98 & 25.91 & - & - & - \\
\hline & $/ \mathbf{i} /$ & 316.94 & 8.31 & 298.34 & 0.07 & 0.02 & 3.17 & 0.85 \\
\hline & $/ \mathbf{u} /$ & 313.14 & 8.90 & 346.39 & 43.50 & 0.03 & 1.06 & 0.47 \\
\hline \multirow{3}{*}{ TV206 } & $/ \mathbf{a} /$ & 680.80 & 14.94 & 610.15 & 59.08 & 0.03 & 1.64 & 0.63 \\
\hline & $/ \mathbf{i} /$ & 313.64 & 20.85 & 398.25 & 16.11 & $<0.01$ & 4.54 & 0.92 \\
\hline & $/ \mathbf{u} /$ & 388.10 & 15.02 & 414.09 & 6.87 & 0.01 & 2.23 & 0.74 \\
\hline \multirow{3}{*}{ TV208 } & $/ \mathbf{a} /$ & 769.06 & 69.36 & 734.54 & 202.51 & 0.69 & 0.23 & 0.11 \\
\hline & $/ \mathbf{i} /$ & 361.78 & 35.79 & 452.75 & 183.59 & 0.23 & 0.69 & 0.33 \\
\hline & $/ \mathbf{u} /$ & 433.31 & 14.80 & 328.43 & 10.67 & $<0.01$ & 8.13 & 0.97 \\
\hline \multirow{3}{*}{ TV209 } & $/ \mathbf{a} /$ & 939.93 & 20.51 & 831.15 & 20.85 & 0.04 & 5.26 & 0.93 \\
\hline & $/ \mathbf{i} /$ & 358.19 & 13.60 & 353.64 & 26.12 & 0.07 & 0.22 & 0.11 \\
\hline & $/ \mathbf{u} /$ & 457.19 & 27.28 & 350.36 & 17.56 & 0.02 & 4.66 & 0.92 \\
\hline \multirow{3}{*}{ TV210 } & $/ \mathbf{a} /$ & 679.39 & 26.12 & 508.56 & 151.34 & 0.03 & 1.57 & 0.62 \\
\hline & $/ \mathbf{i} /$ & 306.07 & 11.27 & 430.50 & 56.42 & $<0.01$ & 3.06 & 0.84 \\
\hline & $/ \mathbf{u} /$ & 414.60 & 7.02 & 443.98 & 150.31 & 0.65 & 0.28 & 0.14 \\
\hline \multirow{3}{*}{ TV211 } & $/ \mathbf{a} /$ & 699.40 & 47.53 & 813.99 & 30.99 & $<0.01$ & 2.86 & 0.82 \\
\hline & $/ \mathbf{i} /$ & 321.53 & 3.75 & 380.96 & 10.68 & $<0.01$ & 7.43 & 0.96 \\
\hline & $/ \mathbf{u} /$ & 378.02 & 13.97 & 358.39 & 27.42 & 0.23 & 0.90 & 0.41 \\
\hline \multirow{3}{*}{ TV301 } & $/ \mathbf{a} /$ & 639.10 & 66.14 & 778.90 & 41.27 & 0.01 & 2.54 & 0.79 \\
\hline & $/ \mathbf{i} /$ & 322.85 & 31.76 & 385.09 & 30.38 & 0.03 & 2.00 & 0.71 \\
\hline & $/ \mathbf{u} /$ & 382.29 & 16.65 & 400.73 & 7.88 & 0.02 & 1.42 & 0.58 \\
\hline \multirow{3}{*}{ TV302 } & $/ \mathbf{a} /$ & 644.15 & 17.53 & 734.45 & 136.71 & 0.16 & 0.93 & 0.42 \\
\hline & $/ \mathbf{i} /$ & 338.38 & 4.06 & 255.23 & 83.67 & 0.06 & 1.40 & 0.57 \\
\hline & $/ \mathbf{u} /$ & 296.34 & 89.44 & 310.18 & 5.74 & 0.72 & 0.22 & 0.11 \\
\hline \multirow{3}{*}{ TV303 } & $/ \mathbf{a} /$ & 759.32 & 39.09 & 726.55 & 63.48 & 0.31 & 0.62 & 0.30 \\
\hline & $/ \mathbf{i} /$ & 347.78 & 2.52 & 323.70 & 43.55 & 0.22 & 0.11 & 0.05 \\
\hline & $/ \mathbf{u} /$ & 371.68 & 19.44 & 377.50 & 26.11 & 0.36 & 0.25 & 0.13 \\
\hline \multirow{3}{*}{ TV304 } & $/ \mathbf{a} /$ & 587.23 & 66.94 & 666.32 & 47.75 & 0.12 & 1.36 & 0.56 \\
\hline & $/ \mathbf{i} /$ & 292.18 & 13.21 & 479.15 & 65.32 & $<0.01$ & 3.97 & 0.89 \\
\hline & $/ \mathbf{u} /$ & 306.52 & 21.84 & 383.64 & 47.25 & 0.01 & 2.10 & 0.72 \\
\hline
\end{tabular}

$\mathrm{SD}=$ Standard Deviation 
Table 4.4 F2 Values by Participant

\begin{tabular}{|c|c|c|c|c|c|c|c|c|}
\hline Part. ID & & $\begin{array}{c}\text { Pre } \\
\text { Mean }\end{array}$ & SD & $\begin{array}{c}\text { Post } \\
\text { Mean }\end{array}$ & SD & p-value & $\begin{array}{c}\text { Cohen's } \\
\text { d }\end{array}$ & $\begin{array}{c}\text { Effect size } \\
\mathbf{r} \\
\end{array}$ \\
\hline \multirow[t]{3}{*}{ TV202 } & $/ \mathbf{a} /$ & - & - & 1115.24 & 83.81 & - & - & - \\
\hline & $/ \mathbf{i} /$ & 1638.02 & 382.56 & 2353.02 & 62.63 & $<0.01$ & 2.61 & 0.79 \\
\hline & $/ \mathbf{u} /$ & 1108.45 & 56.65 & 1243.17 & 89.44 & 0.02 & 1.80 & 0.67 \\
\hline \multirow[t]{3}{*}{ TV206 } & $/ \mathbf{a} /$ & 1203.05 & 24.87 & 1275.62 & 114.18 & 0.14 & 0.88 & 0.40 \\
\hline & $/ \mathbf{i} /$ & 2051.31 & 118.54 & 901.20 & 132.18 & $<0.01$ & 9.16 & 0.98 \\
\hline & $/ \mathbf{u} /$ & 1290.78 & 92.15 & 1212.78 & 137.55 & 0.19 & 0.67 & 0.32 \\
\hline \multirow[t]{3}{*}{ TV208 } & $/ \mathbf{a} /$ & 1248.98 & 63.23 & 1340.29 & 182.42 & 0.32 & 0.67 & 0.32 \\
\hline & $/ \mathbf{i} /$ & 2328.39 & 25.19 & 2250.27 & 26.92 & $<0.01$ & 3.00 & 0.83 \\
\hline & $/ \mathbf{u} /$ & 967.38 & 84.67 & 1093.14 & 115.03 & 0.12 & 1.25 & 0.53 \\
\hline \multirow[t]{3}{*}{ TV209 } & $/ \mathbf{a} /$ & 831.15 & 20.85 & 1358.31 & 63.72 & 0.11 & 11.12 & 0.98 \\
\hline & $/ \mathbf{i} /$ & 2508.58 & 106.09 & 1438.33 & 44.70 & $<0.01$ & 13.15 & 0.99 \\
\hline & $/ \mathbf{u} /$ & 1435.04 & 90.79 & 1332.79 & 18.72 & 0.06 & 1.56 & 0.62 \\
\hline \multirow[t]{3}{*}{ TV210 } & $/ \mathbf{a} /$ & 1190.21 & 66.89 & 944.99 & 334.41 & 0.18 & 1.02 & 0.45 \\
\hline & $/ \mathbf{i} /$ & 2453.20 & 247.39 & 1510.55 & 317.57 & $<0.01$ & 3.31 & 0.86 \\
\hline & $/ \mathbf{u} /$ & 1277.41 & 190.88 & 1153.70 & 139.92 & 0.20 & 0.74 & 0.35 \\
\hline \multirow[t]{3}{*}{ TV211 } & $/ \mathbf{a} /$ & 1500.76 & 49.03 & 1583.59 & 23.35 & 0.02 & 2.16 & 0.73 \\
\hline & $/ \mathbf{i} /$ & 2530.69 & 117.45 & 1765.85 & 39.73 & $<0.01$ & 8.72 & 0.97 \\
\hline & $/ \mathbf{u} /$ & 1462.88 & 63.01 & 1364.64 & 183.70 & 0.16 & 0.72 & 0.34 \\
\hline \multirow[t]{3}{*}{ TV301 } & $/ \mathbf{a} /$ & 1139.13 & 50.93 & 1342.88 & 58.60 & $<0.01$ & 3.71 & 0.88 \\
\hline & $/ \mathbf{i} /$ & 1995.35 & 485.49 & 1639.71 & 338.25 & 0.28 & 0.85 & 0.39 \\
\hline & $/ \mathbf{u} /$ & 1078.54 & 64.01 & 1059.63 & 32.17 & 0.53 & 0.37 & 0.18 \\
\hline \multirow[t]{3}{*}{ TV302 } & $/ \mathbf{a} /$ & 1237.86 & 21.69 & 1297.31 & 131.04 & 0.35 & 0.63 & 0.30 \\
\hline & $/ \mathbf{i} /$ & 2129.09 & 481.61 & 1872.73 & 631.76 & 0.51 & 0.46 & 0.22 \\
\hline & $/ \mathbf{u} /$ & 917.75 & 65.61 & 1160.44 & 16.20 & $<0.01$ & 5.08 & 0.93 \\
\hline \multirow[t]{3}{*}{ TV303 } & $/ \mathbf{a} /$ & 1408.57 & 66.99 & 1357.09 & 53.37 & 0.25 & 0.85 & 0.39 \\
\hline & $/ \mathbf{i} /$ & 977.46 & 356.48 & 832.89 & 72.87 & 0.30 & 0.56 & 0.27 \\
\hline & $/ \mathbf{u} /$ & 1250.27 & 36.59 & 1356.52 & 81.47 & 0.05 & 1.68 & 0.64 \\
\hline \multirow[t]{3}{*}{ TV304 } & $/ \mathbf{a} /$ & 1314.34 & 20.75 & 1297.34 & 15.16 & 0.15 & 0.94 & 0.42 \\
\hline & $/ \mathbf{i} /$ & 2187.91 & 81.46 & 1371.56 & 175.96 & $<0.01$ & 5.95 & 0.95 \\
\hline & $/ \mathbf{u} /$ & 1075.28 & 97.04 & 1333.32 & 69.15 & $<0.01$ & 3.06 & 0.84 \\
\hline
\end{tabular}

$\mathrm{SD}=$ Standard Deviation 
Table 4.5, 4.6, and 4.7 provide summaries of the means for PPQ, RAP, and NHR of vowel prolongations for /a, i, and $\mathrm{u} /$ by participant. The changes in these values varied by participant and vowel. A decrease in these values indicates more regular vocal fold vibratory patterns. These variables were measured during the prolongation of the vowels /a, i, and $\mathrm{u} /$. As a result of technical difficulties, data for the vowel /a/ was not recorded for one participant.

P-values for PPQ and RAP during vowel prolongations were statistically significant for eight out of 30 vowel productions $(p \leq 0.05)$. The vowel $/ a /$ was found to present improved vocal fold vibratory patterns across both measures (PPQ and RAP). Two participants achieved statistically significantly changes in reduced breathiness for the vowel /a/. Results also revealed statistically significant increases in values in two participants for the vowel /i/ and four participants for the vowel /u/. Effect sizes of PPQ and RAP varied by vowel and participant. PPQ analyses for the vowel $/ \mathrm{a} /$ revealed decreased values (pre-training mean $=0.35, \mathrm{SD}=0.17$; posttraining mean $=0.31$, SD 0.13). Cohen's (d) indicated a large effect size for four participants ( $\mathrm{r}=0.61$ to 0.88$)$. There was also a medium effect size for four participants $(r=0.31$ to 0.35$)$. Three samples of $/ \mathrm{a} /$ had low effect sizes $(r=0.02$ to 0.09). Contrary to the findings of the vowel/a/, PPQ and RAP analyses for vowels /i/ and $/ \mathrm{u} /$ indicated an increase in values. For the vowel $/ \mathrm{i} /$, large effect sizes were present in four participants. Three participants had medium effect sizes $(r=0.26$ to 0.29). The remaining three participants, with small effect size values $(\mathrm{r}=<0.04)$. Analyses of RAP effect sizes closely followed the trends of PPQ analyses likely due to the fact that is it also a measure of regularity of vocal fold vibration. 
NHR analyses revealed decreases in values across all participants' pre- and post-training. NHR is a measure of a vocal quality perceptually known as breathiness (Dahl and Mahler, 2019). The norms provided for the Multi-Dimensional Voice Program (MDVP) by Kay Pentax suggested that participants presented with a breathier voice than individuals studied to develop the norms for the participants' age group and gender.

P-values for NHR revealed statistically significant changes for eight out of 29 vowels. Data analyses revealed statistically significantly decreased NHR values for two participants in their production of the vowel /a/, two participants for the vowel /i/, and four participants for the vowel $/ \mathrm{u} /$. The vowel $/ \mathrm{a} /$ contained five participants with large effect sizes ( $r=0.42$ to 0.73$)$, one with a medium effect size, $(r=0.29)$, and one with a small effect size $(r=0.19)$. One participant was found to have no change $(r=$ 0.00). Six participants had large effect sizes $(\mathrm{r}=0.42$ to 0.86$)$ for the vowel $/ \mathrm{i} /$. One participant had a medium effect size $(r=0.26)$, two participants had small effect sizes $(r=0.16$ to 0.19$)$, and one had no effect $(r=0.00)$. For the vowel $/ \mathrm{u} /$, five participants had large effect sizes ( $r=0.43$ to 0.71$)$. Three participants were determined to have medium effect sizes $(r=0.28$ to 0.34$)$. One participant had a small effect size $(r=$ $0.21)$, and one participant had no change $(\mathrm{r}=0.00)$. 
Table 4.5 PPQ Values by Participant

\begin{tabular}{|c|c|c|c|c|c|c|c|c|}
\hline Part. ID & & $\begin{array}{c}\text { Pre } \\
\text { Means } \\
(\%) \\
\end{array}$ & $\mathrm{SD}$ & $\begin{array}{c}\text { Post } \\
\text { Means } \\
(\%)\end{array}$ & SD & P-Value & $\begin{array}{c}\text { Cohen's } \\
\text { d }\end{array}$ & $\begin{array}{c}\text { Effect Size } \\
\mathbf{r}\end{array}$ \\
\hline \multirow{3}{*}{ TV202 } & $/ \mathbf{a} /$ & - & - & 0.51 & 0.26 & - & - & - \\
\hline & $/ \mathbf{i} /$ & 0.02 & 0.00 & 0.49 & 0.28 & 0.21 & 2.36 & 0.76 \\
\hline & $/ \mathbf{u} /$ & 0.02 & 0.00 & 0.60 & 0.17 & 0.02 & 4.88 & 0.93 \\
\hline \multirow{3}{*}{ TV206 } & $/ \mathbf{a} /$ & 0.31 & 0.05 & 0.26 & 0.08 & 0.35 & 0.74 & 0.35 \\
\hline & $/ \mathbf{i} /$ & 0.31 & 0.16 & 0.23 & 0.08 & 0.22 & 0.61 & 0.29 \\
\hline & $/ \mathbf{u} /$ & 0.20 & 0.07 & 0.44 & 0.18 & 0.02 & 1.78 & 0.66 \\
\hline \multirow{3}{*}{ TV208 } & $/ \mathbf{a} /$ & 0.51 & 0.42 & 0.29 & 0.25 & 0.41 & 0.64 & 0.31 \\
\hline & $/ \mathbf{i} /$ & 0.44 & 0.28 & 0.42 & 0.23 & 0.90 & 0.08 & 0.04 \\
\hline & $/ \mathbf{u} /$ & 0.15 & 0.21 & 0.48 & 0.32 & 0.13 & 1.21 & 0.52 \\
\hline \multirow{3}{*}{ TV209 } & $\mid \mathbf{a} /$ & 0.35 & 0.15 & 0.15 & 0.03 & 0.20 & 1.82 & 0.67 \\
\hline & $/ \mathbf{i} /$ & 0.63 & 0.53 & 0.42 & 0.13 & 0.46 & 0.55 & 0.26 \\
\hline & $/ \mathbf{u} /$ & 0.28 & 0.32 & 0.20 & 0.10 & 0.60 & 0.33 & 0.33 \\
\hline \multirow{3}{*}{ TV210 } & $/ \mathbf{a} /$ & 0.02 & 0.00 & 0.35 & 0.16 & $<0.01$ & 2.95 & 0.83 \\
\hline & $/ \mathbf{i} /$ & 0.02 & 0.00 & 0.47 & 0.39 & 0.04 & 1.63 & 0.63 \\
\hline & $/ \mathbf{u} /$ & 0.59 & 0.46 & 0.59 & 0.32 & 1.00 & 0.00 & 0.00 \\
\hline \multirow{3}{*}{ TV211 } & $/ \mathbf{a} /$ & 0.27 & 0.02 & 0.16 & 0.04 & $<0.01$ & 3.64 & 0.88 \\
\hline & $/ \mathbf{i} /$ & 0.52 & 0.28 & 0.17 & 0.05 & 0.02 & 1.77 & 0.66 \\
\hline & $/ \mathbf{u} /$ & 0.33 & 0.05 & 0.15 & 0.08 & 0.01 & 2.87 & 0.82 \\
\hline \multirow{3}{*}{ TV301 } & $/ \mathbf{a} /$ & 0.23 & 0.05 & 0.16 & 0.04 & 0.07 & 1.55 & 0.61 \\
\hline & $/ \mathbf{i} /$ & 0.45 & 0.26 & 0.47 & 0.16 & 0.92 & 0.09 & 0.05 \\
\hline & $/ \mathbf{u} /$ & 0.29 & 0.07 & 0.45 & 0.13 & 0.04 & 1.48 & 0.59 \\
\hline \multirow{3}{*}{ TV302 } & $/ \mathbf{a} /$ & 0.57 & 0.16 & 0.43 & 0.24 & 0.44 & 0.68 & 0.32 \\
\hline & $/ \mathbf{i} /$ & 0.21 & 0.11 & 0.38 & 0.25 & 0.22 & 0.87 & 0.40 \\
\hline & $/ \mathbf{u} /$ & 0.25 & 0.10 & 0.38 & 0.20 & 0.29 & 0.80 & 0.37 \\
\hline \multirow{3}{*}{ TV303 } & $/ \mathbf{a} /$ & 0.38 & 0.15 & 0.39 & 0.27 & 0.98 & 0.05 & 0.02 \\
\hline & $/ \mathbf{i} /$ & 0.33 & 0.39 & 0.36 & 0.39 & 0.90 & 0.08 & 0.04 \\
\hline & $/ \mathbf{u} /$ & 0.35 & 0.13 & 0.44 & 0.23 & 0.59 & 0.48 & 0.23 \\
\hline \multirow{3}{*}{ TV304 } & $/ \mathbf{a} /$ & 0.47 & 0.23 & 0.43 & 0.21 & 0.77 & 0.18 & 0.09 \\
\hline & $/ \mathbf{i} /$ & 0.61 & 0.33 & 0.43 & 0.33 & 0.38 & 0.54 & 0.26 \\
\hline & $/ \mathbf{u} /$ & 0.30 & 0.05 & 0.43 & 0.15 & 0.13 & 1.18 & 0.51 \\
\hline
\end{tabular}

$\mathrm{SD}=$ Standard Deviation 
Table 4.6 RAP Values by Participant

\begin{tabular}{|c|c|c|c|c|c|c|c|c|}
\hline Part. ID & & $\begin{array}{c}\text { Pre } \\
\text { Means } \\
(\%)\end{array}$ & $\mathrm{SD}$ & $\begin{array}{c}\text { Post } \\
\text { Means } \\
(\%)\end{array}$ & SD & P-Value & $\begin{array}{c}\text { Cohen's } \\
\text { d }\end{array}$ & $\begin{array}{c}\text { Effect Size } \\
\mathbf{r}\end{array}$ \\
\hline \multirow{3}{*}{ TV202 } & $/ \mathbf{a} /$ & - & - & 0.53 & 0.27 & - & - & - \\
\hline & $/ \mathbf{i} /$ & 0.02 & 0.00 & 0.51 & 0.30 & 0.21 & 2.31 & 0.76 \\
\hline & $/ \mathbf{u} /$ & 0.02 & 0.00 & 0.62 & 0.22 & 0.05 & 3.82 & 0.89 \\
\hline \multirow{3}{*}{ TV206 } & $/ \mathbf{a} /$ & 0.31 & 0.05 & 0.27 & 0.09 & 0.49 & 0.53 & 0.26 \\
\hline & $/ \mathbf{i} /$ & 0.32 & 0.20 & 0.23 & 0.10 & 0.19 & 0.56 & 0.27 \\
\hline & $/ \mathbf{u} /$ & 0.19 & 0.07 & 0.45 & 0.18 & 0.02 & 1.91 & 0.69 \\
\hline \multirow{3}{*}{ TV208 } & $/ \mathbf{a} /$ & 0.40 & 0.44 & 0.29 & 0.26 & 0.67 & 0.31 & 0.15 \\
\hline & $/ \mathbf{i} /$ & 0.45 & 0.30 & 0.43 & 0.24 & 0.90 & 0.07 & 0.04 \\
\hline & $/ \mathbf{u} /$ & 0.08 & 0.16 & 0.49 & 0.34 & 0.05 & 1.56 & 0.61 \\
\hline \multirow{3}{*}{ TV209 } & $/ \mathbf{a} /$ & 0.35 & 0.16 & 0.16 & 0.06 & 0.26 & 1.56 & 0.62 \\
\hline & $/ \mathbf{i} /$ & 0.59 & 0.49 & 0.43 & 0.13 & 0.52 & 0.44 & 0.22 \\
\hline & $/ \mathbf{u} /$ & 0.30 & 0.35 & 0.24 & 0.14 & 0.67 & 0.22 & 0.11 \\
\hline \multirow{3}{*}{ TV210 } & $/ \mathbf{a} /$ & 0.02 & 0.00 & 0.35 & 0.16 & $<0.01$ & 2.86 & 0.82 \\
\hline & $/ \mathbf{i} /$ & 0.02 & 0.00 & 0.77 & 0.61 & 0.03 & 1.73 & 0.65 \\
\hline & $/ \mathbf{u} /$ & 0.45 & 0.48 & 0.45 & 0.32 & 0.99 & 0.00 & 0.00 \\
\hline \multirow{3}{*}{ TV211 } & $/ \mathbf{a} /$ & 0.27 & 0.03 & 0.17 & 0.04 & $<0.01$ & 2.63 & 0.80 \\
\hline & $/ \mathbf{i} /$ & 0.55 & 0.29 & 0.21 & 0.07 & 0.03 & 1.61 & 0.63 \\
\hline & $/ \mathbf{u} /$ & 0.34 & 0.04 & 0.18 & 0.10 & 0.03 & 2.10 & 0.72 \\
\hline \multirow{3}{*}{ TV301 } & $/ \mathbf{a} /$ & 0.20 & 0.07 & 0.17 & 0.05 & 0.40 & 0.52 & 0.25 \\
\hline & $/ \mathbf{i} /$ & 0.44 & 0.27 & 0.52 & 0.17 & 0.60 & 0.35 & 0.17 \\
\hline & $/ \mathbf{u} /$ & 0.26 & 0.08 & 0.45 & 0.12 & 0.02 & 1.83 & 0.68 \\
\hline \multirow{3}{*}{ TV302 } & $/ \mathbf{a} /$ & 0.57 & 0.17 & 0.54 & 0.31 & 0.77 & 0.12 & 0.06 \\
\hline & $/ \mathbf{i} /$ & 0.24 & 0.13 & 0.41 & 0.28 & 0.22 & 0.79 & 0.37 \\
\hline & $/ \mathbf{u} /$ & 0.28 & 0.12 & 0.41 & 0.23 & 0.32 & 0.71 & 0.33 \\
\hline \multirow{3}{*}{ TV303 } & $/ \mathbf{a} /$ & 0.41 & 0.17 & 0.40 & 0.26 & 0.87 & 0.05 & 0.02 \\
\hline & $/ \mathbf{i} /$ & 0.33 & 0.40 & 0.38 & 0.41 & 0.84 & 0.12 & 0.06 \\
\hline & $/ \mathbf{u} /$ & 0.41 & 0.20 & 0.47 & 0.22 & 0.77 & 0.28 & 0.14 \\
\hline \multirow{3}{*}{ TV304 } & $/ \mathbf{a} /$ & 0.53 & 0.32 & 0.45 & 0.23 & 0.49 & 0.29 & 0.14 \\
\hline & $/ \mathbf{i} /$ & 0.61 & 0.32 & 0.42 & 0.33 & 0.52 & 0.59 & 0.28 \\
\hline & $/ \mathbf{u} /$ & 0.24 & 0.13 & 0.48 & 0.19 & 0.08 & 1.49 & 0.60 \\
\hline
\end{tabular}

$\mathrm{SD}=$ Standard Deviation 
Table 4.7 NHR Values by Participant

\begin{tabular}{|c|c|c|c|c|c|c|c|c|}
\hline Part. ID & & $\begin{array}{c}\text { Pre } \\
\text { Means } \\
\text { (dB SPL) }\end{array}$ & $\mathrm{SD}$ & $\begin{array}{c}\text { Post } \\
\text { Means } \\
\text { (dB SPL) }\end{array}$ & $\mathrm{SD}$ & P-Value & $\begin{array}{c}\text { Cohen's } \\
\text { d }\end{array}$ & $\begin{array}{c}\text { Effect Size } \\
\mathbf{r}\end{array}$ \\
\hline \multirow[t]{3}{*}{ TV202 } & $/ \mathbf{a} /$ & - & - & 0.53 & 0.27 & - & - & - \\
\hline & $/ \mathbf{i} /$ & 0.02 & 0.00 & 0.51 & 0.30 & 0.21 & 2.31 & 0.76 \\
\hline & $/ \mathbf{u} /$ & 0.02 & 0.00 & 0.62 & 0.22 & 0.05 & 3.82 & 0.89 \\
\hline \multirow[t]{3}{*}{ TV206 } & $/ \mathbf{a} /$ & 0.31 & 0.05 & 0.27 & 0.09 & 0.49 & 0.53 & 0.26 \\
\hline & $/ \mathbf{i} /$ & 0.32 & 0.20 & 0.23 & 0.10 & 0.19 & 0.56 & 0.27 \\
\hline & $/ \mathbf{u} /$ & 0.19 & 0.07 & 0.45 & 0.18 & 0.02 & 1.91 & 0.69 \\
\hline \multirow[t]{3}{*}{ TV208 } & $/ \mathbf{a} /$ & 0.40 & 0.44 & 0.29 & 0.26 & 0.67 & 0.31 & 0.15 \\
\hline & $/ \mathbf{i} /$ & 0.45 & 0.30 & 0.43 & 0.24 & 0.90 & 0.07 & 0.04 \\
\hline & $/ \mathbf{u} /$ & 0.08 & 0.16 & 0.49 & 0.34 & 0.05 & 1.56 & 0.61 \\
\hline \multirow[t]{3}{*}{ TV209 } & $/ \mathbf{a} /$ & 0.35 & 0.16 & 0.16 & 0.06 & 0.26 & 1.56 & 0.62 \\
\hline & $/ \mathbf{i} /$ & 0.59 & 0.49 & 0.43 & 0.13 & 0.52 & 0.44 & 0.22 \\
\hline & $/ \mathbf{u} /$ & 0.30 & 0.35 & 0.24 & 0.14 & 0.67 & 0.22 & 0.11 \\
\hline \multirow[t]{3}{*}{ TV210 } & $/ \mathbf{a} /$ & 0.02 & 0.00 & 0.35 & 0.16 & $<0.01$ & 2.86 & 0.82 \\
\hline & $/ \mathbf{i} /$ & 0.02 & 0.00 & 0.77 & 0.61 & 0.03 & 1.73 & 0.65 \\
\hline & $/ \mathbf{u} /$ & 0.45 & 0.48 & 0.45 & 0.32 & 0.99 & 0.00 & 0.00 \\
\hline \multirow[t]{3}{*}{ TV211 } & $/ \mathbf{a} /$ & 0.27 & 0.03 & 0.17 & 0.04 & $<0.01$ & 2.63 & 0.80 \\
\hline & $/ \mathbf{i} /$ & 0.55 & 0.29 & 0.21 & 0.07 & 0.03 & 1.61 & 0.63 \\
\hline & $/ \mathbf{u} /$ & 0.34 & 0.04 & 0.18 & 0.10 & 0.03 & 2.10 & 0.72 \\
\hline \multirow[t]{3}{*}{ TV301 } & $/ \mathbf{a} /$ & 0.20 & 0.07 & 0.17 & 0.05 & 0.40 & 0.52 & 0.25 \\
\hline & $/ \mathbf{i} /$ & 0.44 & 0.27 & 0.52 & 0.17 & 0.60 & 0.35 & 0.17 \\
\hline & $/ \mathbf{u} /$ & 0.26 & 0.08 & 0.45 & 0.12 & 0.02 & 1.83 & 0.68 \\
\hline \multirow[t]{3}{*}{ TV302 } & $/ \mathbf{a} /$ & 0.57 & 0.17 & 0.54 & 0.31 & 0.77 & 0.12 & 0.06 \\
\hline & $/ \mathbf{i} /$ & 0.24 & 0.13 & 0.41 & 0.28 & 0.22 & 0.79 & 0.37 \\
\hline & $/ \mathbf{u} /$ & 0.28 & 0.12 & 0.41 & 0.23 & 0.32 & 0.71 & 0.33 \\
\hline \multirow[t]{3}{*}{ TV303 } & $/ \mathbf{a} /$ & 0.41 & 0.17 & 0.40 & 0.26 & 0.87 & 0.05 & 0.02 \\
\hline & $/ \mathbf{i} /$ & 0.33 & 0.40 & 0.38 & 0.41 & 0.84 & 0.12 & 0.06 \\
\hline & $/ \mathbf{u} /$ & 0.41 & 0.20 & 0.47 & 0.22 & 0.77 & 0.28 & 0.14 \\
\hline \multirow[t]{3}{*}{ TV304 } & $/ \mathbf{a} /$ & 0.53 & 0.32 & 0.45 & 0.23 & 0.49 & 0.29 & 0.14 \\
\hline & $/ \mathbf{i} /$ & 0.61 & 0.32 & 0.42 & 0.33 & 0.52 & 0.59 & 0.28 \\
\hline & $/ \mathbf{u} /$ & 0.24 & 0.13 & 0.48 & 0.19 & 0.08 & 1.49 & 0.60 \\
\hline
\end{tabular}

$\mathrm{SD}=$ Standard Deviation 
Tables 4.8 and 4.9 summarize the mean vocal intensities of each participant during vowel prolongation and spontaneous speech tasks. Mean vocal intensity increased post-training compared with pre-training. All participants produced speech at a gender and age-appropriate intensity level for conversation (Yiu and Yip, 2016).

Two-tailed paired-sample t-tests for this variable revealed statistically significant results $(\mathrm{p} \leq 0.05)$ for 19 out of 24 vowels, and five out of 16 spontaneous speech samples. The researchers found that six participants had statistically significantly increased intensity during the production of the vowel /a/, seven participants had statistically significant increases in intensity during the production of the vowel /i/, and six participants demonstrated statistically significant gains in intensity during the production of the vowel $/ \mathrm{u} /$.

The analysis of spontaneous speech samples resulted in statistically significantly increased intensity for four participants during the picture description task. One participant showed statistically significant increased intensity during the monologue task. Effect sizes for intensity were also calculated for both tasks. Analysis of the picture description task revealed large effect sizes for six participants $(r=0.51$ to 0.78$)$. Two participants produced a medium effect size $(\mathrm{r}=0.28$ to 0.33$)$, and one participant produced a small effect size $(\mathrm{r}=0.14)$. The monologue speech task also demonstrated increases in intensity post-training. Three participants had large effect sizes $(r=0.51$ to 0.46$)$, and one participant had a large effect size $(r=0.42)$. One participant had a medium effect size $(r=0.27)$, and 3 had little effect $(r=0.04$ to 0.09). 
Table 4.8 Intensity of Vowel Prolongation by Participant

\begin{tabular}{|c|c|c|c|c|c|c|c|c|}
\hline Part. ID & & $\begin{array}{c}\text { Pre } \\
\text { Means } \\
\text { (dB } \\
\text { SPL) } \\
\end{array}$ & SD & $\begin{array}{c}\text { Post } \\
\text { Means } \\
\text { (dB } \\
\text { SPL) } \\
\end{array}$ & SD & P-Value & $\begin{array}{c}\text { Cohen's } \\
\text { d }\end{array}$ & $\begin{array}{c}\text { Effect Size } \\
\mathbf{r}\end{array}$ \\
\hline \multirow{3}{*}{ TV202 } & $/ \mathbf{a} /$ & 73.52 & 2.40 & - & - & - & - & - \\
\hline & $/ \mathbf{i} /$ & 75.58 & 1.71 & - & - & - & - & - \\
\hline & $/ \mathbf{u} /$ & 81.33 & 4.25 & - & - & - & - & - \\
\hline \multirow{3}{*}{ TV206 } & $/ \mathbf{a} /$ & 75.38 & 2.16 & 77.58 & 1.44 & $<0.01$ & 1.33 & 0.56 \\
\hline & $/ \mathbf{i} /$ & 74.63 & 1.51 & 78.19 & 1.07 & $<0.01$ & 2.72 & 0.81 \\
\hline & $/ \mathbf{u} /$ & 75.35 & 1.46 & 79.67 & 1.24 & $<0.01$ & 1.31 & 0.55 \\
\hline \multirow{3}{*}{ TV208 } & $/ \mathbf{a} /$ & - & - & 74.80 & 1.47 & - & - & - \\
\hline & $/ \mathbf{i} /$ & - & - & 75.98 & 2.40 & - & - & - \\
\hline & $/ \mathbf{u} /$ & - & - & 78.13 & 1.53 & - & - & - \\
\hline \multirow{3}{*}{ TV209 } & $/ \mathbf{a} /$ & 75.73 & 1.60 & 76.86 & 2.20 & 0.16 & 0.59 & 0.28 \\
\hline & $/ \mathbf{i} /$ & 75.31 & 0.99 & 75.83 & 1.90 & 0.58 & 0.34 & 0.17 \\
\hline & $/ \mathbf{u} /$ & 74.51 & 0.68 & 76.13 & 1.60 & 0.05 & 1.32 & 0.55 \\
\hline \multirow{3}{*}{ TV210 } & $/ \mathbf{a} /$ & 71.44 & 1.18 & 71.86 & 2.19 & 1.00 & 0.00 & 0.00 \\
\hline & $/ \mathbf{i} /$ & 73.03 & 1.01 & 72.05 & 1.72 & 0.03 & 0.00 & 0.00 \\
\hline & $/ \mathbf{u} /$ & 73.61 & 2.68 & 73.99 & 1.85 & 0.24 & 0.00 & 0.00 \\
\hline \multirow{3}{*}{ TV211 } & $/ \mathbf{a} /$ & 73.79 & 1.68 & 75.86 & 3.11 & $<0.01$ & 0.83 & 0.38 \\
\hline & $/ \mathbf{i} /$ & 72.88 & 0.94 & 73.90 & 2.16 & $<0.01$ & 0.61 & 0.29 \\
\hline & $/ \mathbf{u} /$ & 73.83 & 1.05 & 75.09 & 3.05 & $<0.01$ & 0.55 & 0.27 \\
\hline \multirow{3}{*}{ TV301 } & $/ \mathbf{a} /$ & 72.02 & 1.55 & 76.43 & 1.29 & $<0.01$ & 3.09 & 0.84 \\
\hline & $/ \mathbf{i} /$ & 72.50 & 1.66 & 82.23 & 1.61 & $<0.01$ & 5.95 & 0.95 \\
\hline & $/ \mathbf{u} /$ & 72.59 & 1.71 & 84.56 & 1.56 & $<0.01$ & 7.31 & 0.96 \\
\hline \multirow{3}{*}{ TV302 } & $/ \mathbf{a} /$ & 81.65 & 2.33 & 77.66 & 4.22 & $<0.01$ & 1.17 & 0.51 \\
\hline & $/ \mathbf{i} /$ & 73.21 & 1.21 & 76.32 & 1.64 & $<0.01$ & 2.16 & 0.73 \\
\hline & $/ \mathbf{u} /$ & 75.54 & 1.56 & 77.26 & 2.42 & $<0.01$ & 0.84 & 0.39 \\
\hline \multirow{3}{*}{ TV303 } & $/ \mathbf{a} /$ & 69.89 & 0.78 & 70.47 & 1.45 & $<0.01$ & 0.50 & 0.24 \\
\hline & $/ \mathbf{i} /$ & 69.78 & 0.83 & 70.48 & 1.05 & $<0.01$ & 0.74 & 0.35 \\
\hline & $/ \mathbf{u} /$ & 70.00 & 0.87 & 72.56 & 1.01 & $<0.01$ & 2.72 & 0.81 \\
\hline \multirow{3}{*}{ TV304 } & $/ \mathbf{a} /$ & 70.80 & 1.72 & 76.28 & 1.08 & $<0.01$ & 3.82 & 0.89 \\
\hline & $/ \mathbf{i} /$ & 70.34 & 1.46 & 76.52 & 1.06 & $<0.01$ & 4.84 & 0.92 \\
\hline & $/ \mathbf{u} /$ & 70.87 & 1.38 & 77.91 & 0.93 & $<0.01$ & 5.98 & 0.95 \\
\hline
\end{tabular}

$\mathrm{SD}=$ Standard Deviation 
Table 4.9 Intensity of Spontaneous Speech Tasks by Participant

\begin{tabular}{|c|c|c|c|c|c|c|c|c|}
\hline $\begin{array}{c}\text { Part. } \\
\text { ID }\end{array}$ & & $\begin{array}{c}\text { Pre } \\
\text { Means } \\
\text { (dB SPL) }\end{array}$ & SD & $\begin{array}{c}\text { Post } \\
\text { Means } \\
\text { (dB SPL) }\end{array}$ & SD & P-Value & $\begin{array}{c}\text { Cohen's } \\
\text { d }\end{array}$ & $\begin{array}{c}\text { Effect Size } \\
\mathbf{r}\end{array}$ \\
\hline \multirow{2}{*}{ TV202 } & Pic. Desc & 75.71 & 4.59 & - & - & - & - & - \\
\hline & Monologue & 73.68 & 3.52 & - & - & - & - & - \\
\hline \multirow{2}{*}{ TV206 } & Pic. Desc & 74.11 & 2.32 & 78.06 & 2.21 & 0.01 & 1.42 & 0.58 \\
\hline & Monologue & 75.16 & 2.30 & 76.31 & 2.78 & 0.37 & 1.04 & 0.46 \\
\hline \multirow{2}{*}{ TV208 } & Pic. Desc & - & - & 74.93 & 2.70 & - & - & - \\
\hline & Monologue & - & - & 75.49 & 2.09 & - & - & - \\
\hline \multirow{2}{*}{ TV209 } & Pic. Desc & 73.06 & 1.54 & 73.62 & 1.68 & 0.10 & 0.27 & 0.14 \\
\hline & Monologue & 75.84 & 3.26 & 74.60 & 2.13 & 0.09 & 0.01 & 0.01 \\
\hline \multirow{2}{*}{ TV210 } & Pic. Desc & 73.79 & 1.12 & 70.66 & 1.53 & $<0.01$ & 2.50 & 0.78 \\
\hline & Monologue & 72.98 & 1.78 & 71.59 & 2.01 & 0.13 & 0.92 & 0.42 \\
\hline \multirow{2}{*}{ TV211 } & Pic. Desc & 73.11 & 1.74 & 75.33 & 2.69 & 0.06 & 1.18 & 0.51 \\
\hline & Monologue & 72.54 & 0.95 & 75.37 & 2.78 & 0.06 & 1.19 & 0.51 \\
\hline \multirow{2}{*}{ TV301 } & Pic. Desc & 72.90 & 1.35 & 75.98 & 1.38 & $<0.01$ & 2.01 & 0.71 \\
\hline & Monologue & 73.82 & 2.13 & 75.11 & 2.34 & 0.59 & 0.55 & 0.27 \\
\hline \multirow{2}{*}{ TV302 } & Pic. Desc & 73.90 & 1.76 & 74.94 & 2.14 & 0.75 & 0.58 & 0.28 \\
\hline & Monologue & 75.58 & 2.13 & 74.79 & 2.92 & 0.29 & 0.17 & 0.09 \\
\hline \multirow{2}{*}{ TV303 } & Pic. Desc & 70.59 & 0.94 & 71.38 & 0.98 & 0.78 & 0.71 & 0.33 \\
\hline & Monologue & 71.00 & 1.10 & 71.11 & 1.01 & 0.62 & 0.09 & 0.04 \\
\hline \multirow{2}{*}{ TV304 } & Pic. Desc & 70.39 & 2.11 & 73.78 & 1.88 & 0.18 & 1.70 & 0.65 \\
\hline & Monologue & 71.03 & 2.16 & 73.28 & 1.83 & 0.03 & 1.12 & 0.49 \\
\hline
\end{tabular}

$\mathrm{SD}=$ Standard Deviation

Finally, table 4.10 provides a summary of each participant's self-perception of voice immediately pre- and immediately post-training based on results from the Transsexual Voice Questionnaire - Male to Female (TVQMtF). There were seven participants whose TVQMtF scores improved post-training. The TVQMtF uses a Likert Scale for a series of statements to examine the participant's self-perception of their voice and communication experiences. A score of one signifies that the statement rarely occurs, while a score of four indicates that the statement usually or always occurs. Davies and Johnston (2015) divided the TVQMtF into six categories, each of which provide insight into the communicative experiences of trans women. The 
categories include: social interaction, emotional, gender and identity, effort, physical aspects, and pitch.

Seven participants reported improved scores for statements related to social interactions. One participant reported increased scores for statements related to social interactions and one reported no change. Three participants reported increased scores for statements related to emotional aspects of communication post-training. One participant reported increased scores for statements related to gender and identity posttraining. Three participants reported increased scores for statements related to effort required for gendered communication, and one participant reported no change. Four participants reported increased scores for statements related to physical aspects of gendered communication. One participant reported increased scores for statements related to pitch, and one reported no change. 
Table 4.11 TVQmtF Score Sums by Category with Differences Pre- and Post- Training

\begin{tabular}{|c|c|c|c|c|c|c|c|c|}
\hline & & $\begin{array}{c}\text { Overall } \\
\text { Score }\end{array}$ & $\begin{array}{c}\text { Social } \\
\text { Interaction }\end{array}$ & Emotional & $\begin{array}{c}\text { Gender \& } \\
\text { Identity }\end{array}$ & Effort & $\begin{array}{c}\text { Physical } \\
\text { Aspects }\end{array}$ & Pitch \\
\hline \multirow{3}{*}{ TV202 } & Pre & 70.00 & 14.00 & 16.00 & 12.00 & 8.00 & 9.00 & 9.00 \\
\hline & Post & 100.00 & 25.00 & 21.00 & 17.00 & 10.00 & 10.00 & 14.00 \\
\hline & Difference & +30.00 & +11.00 & +5.00 & +5.00 & +2.00 & +1.00 & +5.00 \\
\hline \multirow{3}{*}{ TV206 } & Pre & 80.00 & 17.00 & 18.00 & 16.00 & 8.00 & 7.00 & 11.00 \\
\hline & Post & 90.00 & 20.00 & 20.00 & 15.00 & 10.00 & 10.00 & 12.00 \\
\hline & Difference & +10.00 & +3.00 & +2.00 & -1.00 & +2.00 & +3.00 & +1.00 \\
\hline \multirow{3}{*}{ TV208 } & Pre & 67.00 & 13.00 & 14.00 & 10.00 & 10.00 & 10.00 & 8.00 \\
\hline & Post & 62.00 & 13.00 & 17.00 & 10.00 & 8.00 & 7.00 & 6.00 \\
\hline & Difference & -5.00 & 0.00 & +3.00 & 0.00 & -2.00 & -3.00 & -2.00 \\
\hline \multirow{3}{*}{ TV209 } & Pre & 42.00 & 8.00 & 8.00 & 7.00 & 4.00 & 6.00 & 8.00 \\
\hline & Post & 50.00 & 9.00 & 12.00 & 11.00 & 4.00 & 5.00 & 8.00 \\
\hline & Difference & +8.00 & +1.00 & +4.00 & +4.00 & 0.00 & -1.00 & 0.00 \\
\hline \multirow{3}{*}{ TV210 } & Pre & 92.00 & 19.00 & 20.00 & 17.00 & 11.00 & 11.00 & 13.00 \\
\hline & Post & 53.00 & 12.00 & 11.00 & 6.00 & 7.00 & 8.00 & 8.00 \\
\hline & Difference & -39.00 & -7.00 & -9.00 & -11.00 & -4.00 & -3.00 & -5.00 \\
\hline \multirow{3}{*}{ TV211 } & Pre & 112.00 & 23.00 & 22.00 & 19.00 & 12.00 & 16.00 & 16.00 \\
\hline & Post & 49.00 & 8.00 & 10.00 & 8.00 & 8.00 & 7.00 & 7.00 \\
\hline & Difference & -63.00 & -15.00 & -12.00 & -11.00 & -4.00 & -9.00 & -9.00 \\
\hline \multirow{3}{*}{ TV301 } & Pre & 94.00 & 19.00 & 21.00 & 19.00 & 11.00 & 9.00 & 13.00 \\
\hline & Post & 49.00 & 9.00 & 11.00 & 10.00 & 8.00 & 4.00 & 6.00 \\
\hline & Difference & -45.00 & -10.00 & -10.00 & -9.00 & -3.00 & -5.00 & -7.00 \\
\hline \multirow{3}{*}{ TV302 } & Pre & 107.00 & 27.00 & 23.00 & 20.00 & 10.00 & 10.00 & 14.00 \\
\hline & Post & 47.00 & 10.00 & 10.00 & 8.00 & 6.00 & 6.00 & 6.00 \\
\hline & Difference & -60.00 & -17.00 & -13.00 & -12.00 & -4.00 & -4.00 & -8.00 \\
\hline \multirow{3}{*}{ TV303 } & Pre & 73.00 & 17.00 & 19.00 & 13.00 & 8.00 & 4.00 & 11.00 \\
\hline & Post & 33.00 & 7.00 & 5.00 & 5.00 & 4.00 & 5.00 & 6.00 \\
\hline & Difference & -40.00 & -10.00 & -14.00 & -8.00 & -4.00 & +1.00 & -5.00 \\
\hline \multirow{3}{*}{ TV304 } & Pre & 90.00 & 17.00 & 23.00 & 18.00 & 10.00 & 8.00 & 13.00 \\
\hline & Post & 72.00 & 14.00 & 15.00 & 12.00 & 11.00 & 10.00 & 9.00 \\
\hline & Difference & -18.00 & -3.00 & -8.00 & -6.00 & +1.00 & +2.00 & -4.00 \\
\hline
\end{tabular}


4.2 Research aim 1: Measure acoustic variables of voice and speech related to gender identity immediately before and immediately after six weeks of a transgender voice training protocol.

The first aim of this research sought to identify the impact of a specific sixweek training protocol on voice and speech variables related to gender identity. These variables include: (1) F0 of vowel prolongation, (2) SFF, (3) SFF variation, (4) F1 and F2, (5) PPQ, (6) RAP, (7) NHR, (8) vocal intensity, and (9) self-perception of voice and communication. We conducted paired t-tests to determine whether, on average, there was a change in acoustic variables from pre- to post-training.

Analyses revealed statistically and clinically significant gains, specifically in F0 of vowel prolongation for all participants. There were also clinically significant increases in F0 of vowel prolongation and SFF of spontaneous speech productions in six out of ten participants. Formant frequencies were inconsistent across participants and vowels. Analyses resulted in statistically and clinically significant gains in frequency of F1 in five participants for the prolongation of the vowel /a/, six for the vowel /i/, and six for the vowel /u/. Analyses of F2 revealed statistically and clinically significant increases in frequency of two participants for the prolongation of the vowel /a/, seven for the vowel /i/, and four for the vowel /u/.

PPQ and RAP followed similar patterns, which varied by participant. For the vowel /a/, there were statistically and clinically significantly decreased measures of breathiness for seven out of nine participants. Participants exhibited statistically significantly increased breathiness for prolongation of the vowel /i/ in two out of nine participants, and three out of nine for the prolongation of the vowel /u/. Clinically 
significant results were present in four out of ten participants for the vowel /i/ and six out of ten participants for the vowel /u/. Three participants demonstrated statistically significantly increased values upon analysis of NHR during the prolongation of the vowel /a/ in two out of ten participants, two out of ten for the vowel /i/, and five out of ten for the vowel $/ \mathrm{u} /$. Clinically significant increases in the analyses of NHR were revealed for two out of ten participants during the prolongation of /a/ and one out of ten participants for the vowel /u/. Medium-to-small effect sizes for the prolongation of the vowel /i/ were not deemed clinically significant.

There were statistically significant increases in intensity across the prolongation of all three vowels $(/ \mathrm{a}, \mathrm{i}, \mathrm{u} /)$ for six out of eight participants samples. Four of the six participants who presented statistically significant gains in intensity, also presented with clinically significant gains. Intensity of spontaneous speech varied more than individual vowel prolongations. Three out of eight participants presented statistically significantly increased intensity during the picture description task, and one out of eight resulted in statistically significantly increased measures of intensity during the monologue task. Clinical significance was determined for five out of eight participants during the picture description task, and four out of eight showed clinically significant increases in intensity during the monologue task, post-training.

Analyses of acoustic measures of speech and voice produced statistical and clinical significance within each participant. Given these results, the researchers determined that a specific six-week speech and voice training protocol had statistically and clinically significant effects on trans women's speech and voice. 


\subsection{Determine whether quantitative changes in acoustic features related to gender}

identity relate to perceptual measures of voice and speech.

The TVQMtF was administered to each participant during the initial interview and exit interview. Self-scoring was analyzed utilizing the following six categories provided by Davies and Johnston (2015); (1) social interaction, (2) emotional components of voice identity, (3) gender and identity, (4) effort in producing a desired voice, (5) physical aspects of voice production, and (6) pitch. A decrease within a specific subtest score indicates greater satisfaction with communicative experiences. A higher score indicates increased difficulty in that area of communication. The overall scores of the TVQMtF indicated improved self-perception of voice for the majority of participants. The mean difference for participants was $-22.2(\mathrm{SD}=31.97)$. Three TVQ sub scores increased from pre- to post-training. The mean differences in the scores of each category are provided in table 4.11. Given these results, the researchers have determined that changes in acoustic features of speech and voice have an affirmative effect on the self-perception of the communicative experiences of trans women.

Table 4.11 Mean TVQMtF differences by category

\begin{tabular}{cccccccc}
\hline & $\begin{array}{c}\text { Overall } \\
\text { TVQ Score }\end{array}$ & $\begin{array}{c}\text { Social } \\
\text { Interaction }\end{array}$ & Emotional & $\begin{array}{c}\text { Gender \& } \\
\text { Identity }\end{array}$ & Effort & $\begin{array}{c}\text { Physical } \\
\text { Aspects }\end{array}$ & Pitch \\
\hline Mean & $\mathbf{- 2 2 . 2 0}$ & $\mathbf{- 4 . 7 0}$ & $\mathbf{- 5 . 2 0}$ & $\mathbf{- 4 . 9 0}$ & $\mathbf{- 1 . 6 0}$ & $\mathbf{- 1 . 8 0}$ & $\mathbf{- 3 . 4 0}$ \\
SD & 31.97 & 8.71 & 7.73 & 6.40 & 2.59 & 3.71 & 4.40 \\
\hline
\end{tabular}

$\mathrm{SD}=$ Standard Deviation 


\subsection{Summary of results}

The results of the current study revealed that a specific six-week training protocol resulted in statistically significant changes in acoustic variables of speech and voice that were clinically significant based on large effect sizes. Average F0 of vowels and SFF during the picture description task presented more gender-congruent characteristics post-training for all participants. One participant resulted in decreased SFF. Other variables, such as PPQ, RAP, NHR, and intensity indicated little statistical significance. There were, however, clinically significant changes, they are less consistent than that of the previously mentioned variables. PPQ and RAP were calculated to have varying degrees of effect size, which depended on the participant. The majority of samples exhibited an increase in PPQ and RAP, which indicated greater variation in vocal fold vibratory patterns. Approximately 53 percent of participants presented with a large effect size for decreased values of NHR. Improved NHR values indicates more harmonic speech. Increased intensity values were identified in most participants during the analysis of spontaneous speech post-training, with only one sample found to have decreased intensity.

The TVQMtF provided insight into gender communication experiences of each participant. Mean differences in pre- and post-training scores indicated improvement in all categories. Although all participants demonstrated gains in F0 of vowel prolongation and SFF, 30 percent of participants reported total TVQMtF scores higher on the post-training questionnaire than pre-training. 


\section{Chapter 5}

\section{DISCUSSION}

The primary purpose of this study was to investigate the effect of a specific six-week speech and voice training protocol for trans women on acoustic and perceptual characteristics of voice and speech. It also sought to assess whether there was a relationship between perceptual self-ratings of speech and voice and acoustic measures. Results indicated that there was a statistically and clinically significant effect of training on acoustic variables of trans women. Outcomes also revealed that scores of self-perception improved post-training. These results are consistent with previous studies that demonstrated a beneficial impact of behavioral voice training for trans women. Results are examined with regard to past research, future planning, and clinical decision-making in the following sections.

\subsection{Aim 1 Hypothesis: Acoustic variables will more closely align with cisgender} women post-training.

This study found that a specific six-week voice training protocol had a statistically and clinically significant impact on frequency across all participants. This finding is based on the p-values and effect sizes between the pre- and post-training data analyses of F0 of vowel prolongation (mean effect size $r=0.89$ ) and SFF of spontaneous speech samples (mean effect size $r=0.31$ ). Past studies have identified changes in the following acoustic variables; SFF, jitter (PPQ and RAP), formants (F1, F2, F3), self-perception of speech and voice, and gender perception by unfamiliar 
listeners (Dahl and Mahler, 2019; Gallena, Stickles, and Stickles, 2017; GorhamRowan and Morris 2006; Hancock, 2016). The present findings provide further evidence that a specific six-week voice training protocol had a statistically and clinically significant effect on acoustic and perceptual features of speech and communication. One of the implications of these findings is that a speech language pathologist should incorporate expectations of the client into setting goals and not focus solely on acoustic features of speech and voice during training. It is vital to address expectations regarding results of training, and to establish realistic goals with each client.

Gelfer and Tice (2012) studied five trans women and identified an increase in SFF following participants' training. Their study, however, did not specify a protocol for training, and maintained focus on the perception of gender based on voice. Gelfer and Tice (2012) only conducted group sessions with participants in their study. This differed from the current study's approach because each participant received two oneon-one training sessions and one group session per week. Based on the differences in the research and the current study, dosage is a variable that is likely to have an impact on outcomes of speech and voice training.

There were large effect sizes for the most salient acoustic features of voice. Analyses of F0 of vowel prolongations revealed consistent increases in the posttraining analyses. Gains identified in SFF, post-training, support the first hypothesis. F1 also indicated statistically and clinically significant gains in eight out of ten participants. In addition, nine out of ten participants demonstrated statistically and clinically significant gains in F2. Those who did not show improvement, revealed 
regression of $0.3 \%-10 \%$ decreases in formant frequency in post-training analyses. Anterior carriage of the tongue, and increased lip spreading are known methods of modifying the vocal tract to alter formants (Quinn and Swain, 2018). While these practices were discussed in training sessions, decreases in F1 and F2 may be the result of various circumstances. One possible explanation is familiarity and comfort within the sound booth and evaluation methods, post-training. This study derived values of F1 and F2 from sustained vowel phonation rather than spontaneous speech tasks, which may have influenced the findings of this research.

PPQ and RAP are measures which analyze the vibratory patterns of the vocal folds. Changes in PPQ and RAP post-training evaluations showed generally increased values, with the exception of the vowel /a/. PPQ and RAP values for /a/ were found to be lower in post-training samples. It is possible that /a/ resulted in decreased values because it is a lax vowel, and requires little tension on the articulators, which allows the participants to focus more on sound production at the level of the vocal folds (Shrivastav, et al., 2011). The vowels /i/ and /u/ require manipulation of the glottal wave as it travels through the vocal tract, demanding more focus on the articulators that are required to create each sound. Loss of focus on voice production could result in increased jitter values (Shrivastav, et al., 2011). NHR measures the voice quality perceptually known as breathiness. Post-training evaluations revealed generally increased NHR values. Increases in NHR indicate reduced harmonic sound as a result of changes in the patterns of vocal fold adduction. Changes in adduction patterns may be the result of structural changes (i.e., vocal nodules) or other factors (i.e., fatigue, dehydration, etc.) (Kang et. al., 2018). In the case of this study, voice training may 
also be an underlying factor of increased NHR due to physiological changes in vocal production. In an attempt to rule out other factors, clinicians provided education regarding vocal hygiene to each participant. These findings are a novel product of the research because there have been no findings reported in regard to changes in NHR as a result of voice training.

5.2 Aim 2 Hypothesis: As acoustic variables more closely align with cisgender women post-training, voice satisfaction will improve.

This study also found a significant effect on participants' self-perception of voice and speech post-training. The TVQMtF provided insight into the communicative experiences of ten trans individuals. Participants' overall scores indicated gains (mean scores decreased from 82.70 to 60.50 ). Three participants reported an increase in scores (increases of $+11.00,+3.00$, and +1.00 ). An increase in score indicates a negative impact on communicative experiences. It is possible that, as a secondary result of training, these scores have increased due to raised self-awareness. As participants progressed through the six-week program, they may learn about what aspects of their communication increase the likelihood of being identified as their desired gender. This increased self-awareness may provide opportunities for participants to form negative feelings toward the self-perception of their communicative abilities. Each subcategory of the TVQMtF, developed by Davies and Johnston (2015), was individually analyzed to determine which aspects of communication demonstrated the most significant changes. There was no 
categorization schema recommended for severity (i.e., mild, moderate, severe) of this questionnaire.

Participants exhibited the greatest gains in emotional aspects of voice and communication (mean scores decreased from 18.4 to 13.2). Four participants reported increased scores. This subcategory involved statements such as, "I feel anxious when I know I have to use my voice," and "I feel discriminated against because of my voice." The six-week training protocol may have had an effect which allowed participants to feel confident in their communicative abilities, thus, improving emotion toward communication skills.

The gender and identity subcategory also demonstrated overall gains (mean scores decreased from 15.10 to 10.20$)$ post-training. Two participants reported an increase in scores (increases of +4.00 and +5.00 ). The gender and identity subcategory included questions such as "My voice makes me feel less feminine than I would like," and "My voice doesn't match my physical appearance." Improvement in scores of this subcategory support an idea that voice training allows participants to practice and experiment with their voice in a safe, judgement-free place (Davies and Johnston, 2015). This, in theory, will provide opportunities for the trans woman to become more comfortable and attuned to her gender identity post-training.

Social interaction is likely an area of concern for many trans individuals. Gains in this subcategory were the most inconsistent among participants (mean scores decreased from 17.40 to 12.70 ). Three participants reported increased scores (increases of $+11.00,+3.00$, and +1.00$)$ and one participant reported no change $(+/-$ 0.00). These findings are particularly interesting because societal norms, form 
expectations to which everyone is expected to subscribe. The manner of which social interactions take place are shaped by learning from experiences within society.

Stigmas are formed by those who express distain for individuals who go against the norm (Levitt, 2019). It is possible that these scores were determined by each participant's individual experiences with social norms or interaction, hence the variation in scores.

Improvement in the subcategory of pitch were medial in terms of subcategory standing (mean scores decreased from 11.6 to 8.2). Two participants reported increased scores (increases of +5 and +1 ), and one participant reported no change in score. The results of this subcategory reveal that participants showed an increased satisfaction in their production of a pitch produced in a range that society considers to be more feminine. These findings are especially interesting because pitch is possibly the most salient perceptual variable in speech (Davies and Johnston, 2015), it was surprising to learn that many participants reported less significant differences than in other subcategories. Statements in the TVQMtF associated with this subcategory included, "The pitch of my speaking voice is too low," and "The pitch of my voice is unreliable." Perceptually, for individuals who are unfamiliar with voice production, it is difficult to distinguish between which aspects of phonation (i.e., larynx, vocal tract, etc.) are the most prominent in pitch. So, one may assume that while answering these questions, the participants in this study have a superficial understanding of the term 'pitch' (Davies and Johnston, 2015).

The final two subcategories, physical aspects of speech communication and effort, appeared to be closely related. Questions included, for example, "I use a great 
deal of effort to produce my voice," and "My voice gets tired quickly." Similarly, mean differences only varied by 0.20 (mean change in effort score $=-1.60$, mean change in physical aspects score $=-1.80$ ). Self-monitoring of pitch, pitch variation, intonation, etc. are essential characteristics for gendered communication. This suggests that constant speech and voice monitoring are physical aspects of communication which require a great deal of mental effort, which is not an area specified in the TVQMtF (Davies and Johnston, 2015). Effort and physical aspects of

communication are both significant factors when examining the effectiveness of training. Individuals without the capacity to maintain physical and/or mental effort of vocal exercises may not be viable candidates for training.

\subsection{Limitations}

Results of this study are based on a small sample size. With regard to the probability of a type I error (false positive), this study is most reliable in identifying large effect sizes between pre- and post-training data for fundamental frequency. This study could not detect causation, so conclusions regarding what aspects of the training caused changes in each variable cannot be made.

This study sought to measure the effect of a specific six-week speech and voice training protocol for trans women. This study was a multiple single-subject design in which participants served as their own control. Nevertheless, the findings of this study may not relate to individuals who subscribe to another gender identity, including trans men and gender-nonconforming persons. 
This study did not assess correlations between acoustic features of speech and voice and perception of gender by unfamiliar listeners. It also did not take into account participants' education level, percentage of time that participants present as their true gender, or access to support (i.e., family involvement, medical services, etc.) (Dahl and Mahler, 2019; Duthie, 2018). These factors could promote carry-over of gains achieved during training and should be included in future studies.

Speech samples were analyzed during several tasks; (1) vowel prolongations (/a, i, and u/), (2) spontaneous speech productions of picture description and monologue tasks. While vowel productions provide a great deal of insight into the acoustic characteristics of an individual's voice, it does not measure other aspects of speech, which contribute to gendered communication. (Quinn and Swain, 2018). In this study, spontaneous speech samples were useful for measuring changes in pitch during a less structured task. However, it is not representative of conversational speech, which is likely to vary more than describing a picture or answering a single question for a minute of sustained monologue (Podesva and Callier, 2015).

Self-perception of communication abilities is highly subjective. Many participants knew little-to-nothing about voice production before training. Through participant education, some participants may have become more critical of their voice, which may have some influence on the scores reported by participants. For future research, additional ratings and scales, such as the Census Auditory-Perceptual Evaluation of Voice (CAPE-V), and Grade, Roughness, Breathiness, Asthenia, Strain scale (GRBAS) may be beneficial as a supplement to the TVQMtF(Hancock and Garabedian, 2013). Correlations between individual TVQMtF scores and individual 
acoustic variables may provide insight into the effect of training on individual participants. It may also be beneficial to clinicians to develop a questionnaire for family or loved ones, to further understand how participants' speech and voice impact home life. It also allows participants' family members or significant others to evaluate communicative abilities outside training rooms and provide a clearer picture of how participants use their voice in activities of daily living.

Lastly, this study performed quantitative acoustic analyses of trans women's voices and compared the results with qualitative information regarding participant's perceptions of their voices. However, this study did not consider the different experiences of each individual. Lack of insight into individual cultural norms, and other societal and linguistic elements may have some effect on the outcomes of this study. Additional research is necessary to further understand how these factors affect acoustic characteristics of speech and voice, and self-perception. 


\section{Chapter 6}

\section{CONCLUSIONS}

This study examined the effect of a specific six-week training protocol for trans women on speech, voice, and self-perception of communication. The results revealed that training had statistically and clinically significant effects on several variables associated with gendered communication. Voice training had the greatest influence on fundamental frequency of vowel production and spontaneous speech during picture description and monologue tasks. This study also found that a specific six-week training protocol also had a significant effect on self-perception of aspects of gendered communication. Training appeared to increase self-confidence in most participants, but confidence is difficult to quantify from a self-reported questionnaire

alone. Overall, mean speaking fundamental frequency, maximum pitch, and variation of intonation were determined to be appropriate targets in an evidence-based voice training program for trans women.

Inconsistency in results of vowel formants and acoustic measures of vocal fold vibration and breathiness suggest that targeting these variables did not produce statistically significant changes toward greater female gender identity, but effect size analyses supported strong clinical significance. Previously published studies identified that addressing pitch is vital to gendered communication (Gelfer and Schofield, 2000). These studies, however, did not provide in-depth descriptions of the protocol implemented. Additional research is necessary to aid in treatment planning and clinical decision making for speech language pathologists. 
As a whole, the goal of any therapy or training is to provide the most effective, evidence-based practice. This is an ongoing process in which clinicians should continually seek knowledge about how to best serve their clients. Societal acceptance of a person who lives outside the established norms is vital to the existence of the LGBTQ+ community. Self-acceptance is an internal challenge that each individual must face, though every person's experience is unique. As the cultural norms begin to evolve to be more open and accepting, it the speech language pathologist's responsibility to recognize the communicative needs of this growing community. The results of this study contribute data to identifying possible training targets for trans women. Further research is needed to examine the most effective speech and voice training strategies and tools to evaluate training outcomes. 


\section{BIBLIOGRAPHY}

Becker, L. (2000). Effect Size (ES) | Effect Size Calculators. https://www.uccs.edu/lbecker/effect-size

Bodoin, E., Byrd, C. and Adler, R. (2014). The Clinical Profile of the Male-to-Female Transgender Person of the 21st Century. Contemporary Issues in Communication Science and Disorders, 41(Spring), pp.39-54. https://doi.org/10.1044/cicsd_41_S_39

Casado, J., Rodríguez-Parra, M. and Adrián, J. (2016). Voice feminization in male-tofemale transgendered clients after Wendler's glottoplasty with vs. without voice therapy support. European Archives of Oto-Rhino-Laryngology, 274(4), pp.2049-2058. https://doi.org/10.1007/s00405-016-4420-8

Clark, Carly J., (2016) Voice and Communication Therapy for the Transgender or Transsexual Client: Service Delivery and Treatment Options. Graduate Independent Studies - Communication Sciences and Disorders. 2. https://ir.library.illinoisstate.edu/giscsd/

Dahl, K. and Mahler, L. (2019). Acoustic Features of Transfeminine Voices and Perceptions of Voice Femininity. Journal of Voice. https://doi.org/10.1016/j.jvoice.2019.05.012

Dejonckere, P., Martens, J., Versnel, H. and Moerman, M. (2007). The Effect of Visible Speech on Perceptual Rating of Pathological Voices, and on Correlation Between Perception and Acoustics. Archives Of OtolaryngologyHead \& Neck Surgery, 133(2), 178. https://10.1001/archotol.133.2.178

Desuter, G., et al. "Voice Outcome Indicators for Unilateral Vocal Fold Paralysis Surgery: a Review of the Literature." European Archives of Oto-RhinoLaryngology, vol. 275, no. 2, 2017, pp. 459-468. https://doi.org/10.1007/s00405-017-4844-9

Duthie, K. (2019). Ethical considerations for health leaders responsible for the care of transgender patients. Healthcare Management Forum, 32(2), pp.113-115. https://doi.org/10.1177/0840470418817481

Gallena, S., Stickels, B. and Stickels, E. (2018). Gender Perception After Raising Vowel Fundamental and Formant Frequencies: Considerations for Oral Resonance Research. Journal of Voice, 32(5), pp.592-601. https://doi.org/10.1016/j.jvoice.2017.06.023

Gelfer, M. and Schofield, K. (2000). Comparison of acoustic and perceptual measures 
of voice in male-to-female transsexuals perceived as female versus those perceived as male. Journal of Voice, 14(1), pp.22-33. https://doi.org/ 10.1016/s0892-1997(00)80092-2

Gelfer, M. and Tice, R. (2013). Perceptual and Acoustic Outcomes of Voice Therapy for Male-to-Female Transgender Individuals Immediately After Therapy and 15 Months Later. Journal of Voice, 27(3), pp.335-347. http://dx.doi.org/10.1016/j.jvoice.2012.07.009

Gorham-Rowan, M. and Morris, R. (2006). Aerodynamic Analysis of Male-to-Female Transgender Voice. Journal of Voice, 20(2), pp.251-262. https://doi.org/10.1016/j.jvoice.2005.03.004

Hancock, A. and Garabedian, L. (2012). Transgender voice and communication treatment: a retrospective chart review of 25 cases. International Journal of Language \& Communication Disorders, 48(1), pp.54-65. https://doi.org/10.1111/j.1460-6984.2012.00185.x

Hardy, T., Boliek, C., Wells, K., Dearden, C., Zalmanowitz, C. and Rieger, J. (2016). Pretreatment Acoustic Predictors of Gender, Femininity, and Naturalness Ratings in Individuals with Male-to-Female Gender Identity. American Journal of Speech-Language Pathology, 25(2), pp.125-137. https://doi.org/10.1044/2015_AJSLP-14-0098

Kang, Young Ae, et al. Detection of Voice Changes Due to Aspiration via Acoustic Voice Analysis. Auris Nasus Larynx, vol. 45, no. 4, 2018, pp. 801-806. https://doi.org/0.1016/j.anl.2017.10.007

Kawitzky, D. and McAllister, T. (2020). The Effect of Formant Biofeedback on the Feminization of Voice in Transgender Women. Journal of Voice, 34(1), 5367. https://doi.org/10.1016/j.jvoice.2018.07.017

King, R., Brown, G., and McCrea, C. (2012). Voice Parameters That Result in Identification or Misidentification of Biological Gender in Male-to-Female Transgender Veterans. International Journal of Transgenderism, 13(3), 117130. https://doi.org/10.1080/15532739.2011.664464

Kumar, R., Banumathy, N., Sharma, P., \& Panda, N. (2019). Normalization of voice parameters in patients with unilateral vocal fold palsy: is it realistic? The Journal of Laryngology \& Otology, 133(12), 1097-1102. https://doi.org/10.1017/S0022215119002494

Lara, P. and Peterson, D. (2018). A Collaborative Effort for Development of a Voice 
Modification Clinic for Transgender Individuals in a Medically Underserved Community. Perspectives of the ASHA Special Interest Groups, 3(14), pp.8794. https://doi.org/10.1044/persp3.SIG14.87

Levitt, Heidi M. A Psychosocial Genealogy of LGBTQ+ Gender: An Empirically Based Theory of Gender and Gender Identity Cultures. Psychology of Women Quarterly, vol. 43, no. 3, 2019, pp. 275-297. https://doi.org/10.1177/0361684319834641

Oates, J. and Dacakis, G. (2015). Transgender Voice and Communication: Research Evidence Underpinning Voice Intervention for Male-to-Female Transsexual Women. Perspectives on Voice and Voice Disorders, 25(2), p.48. https://doi.org/10.1044/vvd25.2.48

Podesva, R., and Callier, P. (2015). Voice Quality and Identity. Annual Review Of Applied Linguistics, 35, 173-194. https://doi.org/10.1017/S0267190514000270

Quinn, S. and Swain, N. (2018). Efficacy of intensive voice feminization therapy in a transgender young offender. Journal of Communication Disorders, 72, pp.115. https://doi.org/10.1016/j.jcomdis.2018.02.001

Sheskin, D. (2003). Handbook of parametric and nonparametric procedures (3rd ed.). Boca Raton: Chapman \& Hall/CRC.

Shrivastav, Rahul, et al. (2011). A Model for the Prediction of Breathiness in Vowels. The Journal of the Acoustical Society of America, vol. 129, no. 3, pp. 16051615. https://doi.org/10.1121/1.3543993

World Professional Association for Transgender Health. (2012). Standards of care for the health of transsexual, transgender, and gender-nonconforming people. https://www.wpath.org/publications/soc

Wagner, I., Fugain, C., Monneron-Girard, L., Cordier, B. and Chabolle, F. (2003). Pitch-Raising Surgery in Fourteen Male-to-Female Transsexuals. The Laryngoscope, 113(7), pp.1157-1165. https://doi.org/10.1097/00005537200307000-00011

Yiu, Edwin M-L., and Priscilla P. Yip. S. (2016). "Effect of Noise on Vocal Loudness and Pitch in Natural Environments: An Accelerometer (Ambulatory Phonation Monitor) Study." Journal of Voice, vol. 30, no. 4, pp. 389-393.

http://dx.doi.org/10.1016/j.jvoice.2015.05.016 